\title{
Modular constraints on conformal field theories with currents
}

\author{
Jin-Beom Bae, Sungjay Lee and Jaewon Song \\ Korea Institute for Advanced Study, \\ 85 Hoegiro, Dongdaemun-Gu, Seoul 02455, South Korea \\ E-mail: kastalean4@gmail.com, sjlee@kias.re.kr, jws611@gmail.com
}

ABSTRACT: We study constraints coming from the modular invariance of the partition function of two-dimensional conformal field theories. We constrain the spectrum of CFTs in the presence of holomorphic and anti-holomorphic currents using the semi-definite programming. In particular, we find the bounds on the twist gap for the non-current primaries depend dramatically on the presence of holomorphic currents, showing numerous kinks and peaks. Various rational CFTs are realized at the numerical boundary of the twist gap, saturating the upper limits on the degeneracies. Such theories include Wess-Zumino-Witten models for the Deligne's exceptional series, the Monster CFT and the Baby Monster CFT. We also study modular constraints imposed by $\mathcal{W}$-algebras of various type and observe that the bounds on the gap depend on the choice of $\mathcal{W}$-algebra in the small central charge region.

KeYwords: Conformal and W Symmetry, Field Theories in Lower Dimensions, Nonperturbative Effects

ArXiv EPRINT: 1708.08815 


\section{Contents}

1 Introduction $\quad 1$

2 Preliminaries $\quad 5$

2.1 Modular differential equation 5

$\begin{array}{lll}2.2 & \text { Deligne's exceptional series and monsters } & 7\end{array}$

$\begin{array}{lll}2.3 & \text { Character of } \mathcal{W} \text {-algebra } & 10\end{array}$

3 Modular constraint with Virasoro algebra $\quad 13$

$\begin{array}{ll}3.1 \text { The modular bootstrap equation } & 13\end{array}$

$\begin{array}{lll}3.2 & \text { Numerical bounds on spectrum gap } & 15\end{array}$

$\begin{array}{llr}4 & \text { Spectroscopy } & 18\end{array}$

$\begin{array}{lll}\text { 4.1 Spectrum analysis in modular bootstrap } & 18\end{array}$

4.2 WZW models with Deligne's exceptional series 21

$4.3 c \geq 8$ RCFTs without Kac-Moody symmetry 26

$5 \quad$ Bootstrapping with $\mathcal{W}$-algebra 32

5.1 Numerical bounds 32

$\begin{array}{lll}5.2 & \text { Spectral analysis } & 34\end{array}$

$\begin{array}{lll}5.3 & \text { Accumulation of the spectrum } & 37\end{array}$

\section{Introduction}

Conformal field theories (CFT) are highly constrained by underlying symmetry. For the case of two-dimensional CFTs, the modular invariance and crossing symmetry put strong constraints on the spectrum and operator product expansion (OPE) coefficients. Especially, the infinite dimensional Virasoro symmetry makes it possible to have CFTs with a finite number of primary operators. Those CFTs are called as rational CFTs $[1,2]$ and it has been known that they can be completely solved using the crossing symmetry constraints when $c<1$.

The full classification of the unitary two-dimensional CFTs is however still out of reach. For the CFTs with $c>1$ (without any extended chiral algebra) there is an infinite number of primary operators. For such theories, modular invariance and crossing symmetry were not enough to completely solve the theory. Nevertheless, in recent years, applying the universal constraints originated from the conformal symmetry and unitarity, a.k.a conformal bootstrap has produced many fruitful results for the higher-dimensional CFTs $[3,4]$ and two-dimensional CFTs with $c>1[5-10]$. 
In the current paper, we further study the consequences of modular invariance for the two-dimensional CFTs with $c>1$, along the line of [11-14]. See also [15-19]. ${ }^{1}$ The previous works mostly focused on the case without extended chiral algebra. Instead, we concentrate on the case of CFTs with holomorphic/anti-holomorphic (higher-spin) currents and also with $\mathcal{W}$-algebra symmetry. We first assume that there are conserved currents of conformal weights $(h, \bar{h})=(j, 0)$ and $(h, \bar{h})=(0, j)$. Under this assumption, we investigate the gap in the twist $\Delta_{t} \equiv \Delta-|j|=2 \min (h, \bar{h})$ for the non-current operators. We observe rather dramatic consequences for the upper bounds on the twist gap for the non-current operators. Also, we explore modular constraints for the CFTs with $\mathcal{W}$-algebra symmetry. We focus on the $\mathcal{W}$-algebra associated with a simple Lie algebra $\mathfrak{g}$, which we denote as $\mathcal{W}(\mathfrak{g})$. We find that the numerical upper bounds depend on the choice of $\mathcal{W}(\mathfrak{g})$-algebra when the central charge is small, namely $c \lesssim \operatorname{rank}(\mathfrak{g})$. For the case of $\mathcal{W}\left(A_{2}\right)$-algebra, this problem has been recently discussed in [23, 24].

The torus partition function of an arbitrary $c>1$ CFT admits a character decomposition of the form

$$
\begin{aligned}
Z(\tau, \bar{\tau})= & \chi_{0}(\tau) \bar{\chi}_{0}(\bar{\tau})+\sum_{h, \bar{h}} d_{h, \bar{h}} \chi_{h}(\tau) \bar{\chi}_{\bar{h}}(\bar{\tau}) \\
& +\sum_{j=1}^{\infty}\left[d_{j} \chi_{j}(\tau) \bar{\chi}_{0}(\bar{\tau})+\tilde{d}_{j} \chi_{0}(\tau) \bar{\chi}_{j}(\bar{\tau})\right]
\end{aligned}
$$

where $\chi_{h}(\tau)$ denotes the Virasoro character for the primary operator of weight $h$. Here the degeneracies $d_{j}, \tilde{d}_{j}, d_{h, \bar{h}}$ have to be non-negative integers. The invariance of (1.1) under $T$-transformation forces the given CFT to have states of integer spin $j=|h-\bar{h}|$ while the invariance under $S$-transformation implies

$$
Z(\tau, \bar{\tau})=Z\left(-\frac{1}{\tau},-\frac{1}{\bar{\tau}}\right)
$$

Combining (1.1) and (1.2), we obtain a constraint

$$
\mathcal{Z}_{0}(\tau, \bar{\tau})+\sum_{j=1}^{\infty}\left[d_{j} \mathcal{Z}_{j}(\tau, \bar{\tau})+\tilde{d}_{j} \mathcal{Z}_{\bar{j}}(\tau, \bar{\tau})\right]+\sum_{h, \bar{h}} d_{h, \bar{h}} \mathcal{Z}_{h, \bar{h}}(\tau, \bar{\tau})=0
$$

where the function $\mathcal{Z}_{\lambda}(\tau, \bar{\tau})$ is defined as $\chi_{\lambda}(\tau) \bar{\chi}_{\lambda}(\bar{\tau})-\chi_{\lambda}\left(-\frac{1}{\tau}\right) \bar{\chi}_{\lambda}\left(-\frac{1}{\bar{\tau}}\right)$. We call (1.3) as the modular bootstrap equation.

We utilize the semi-definite programming with the help of powerful numerical package SDPB [26] to examine the modular bootstrap equation (1.3). The semi-definite programming method has been first employed in [12] and then further refined in [14], leading to universal constraints on the CFT spectrum. For example, the upper bound on the spin-independent gap $\Delta_{\text {gap }}$ in the operator dimension is given as

$$
\frac{1}{12} \leq \lim _{c \rightarrow \infty} \frac{\Delta_{\text {gap }}}{c}<\frac{1}{9} .
$$

\footnotetext{
${ }^{1}$ Very recently, constraints on 2d CFT from the genus two partition function have been studied [20-22].
} 


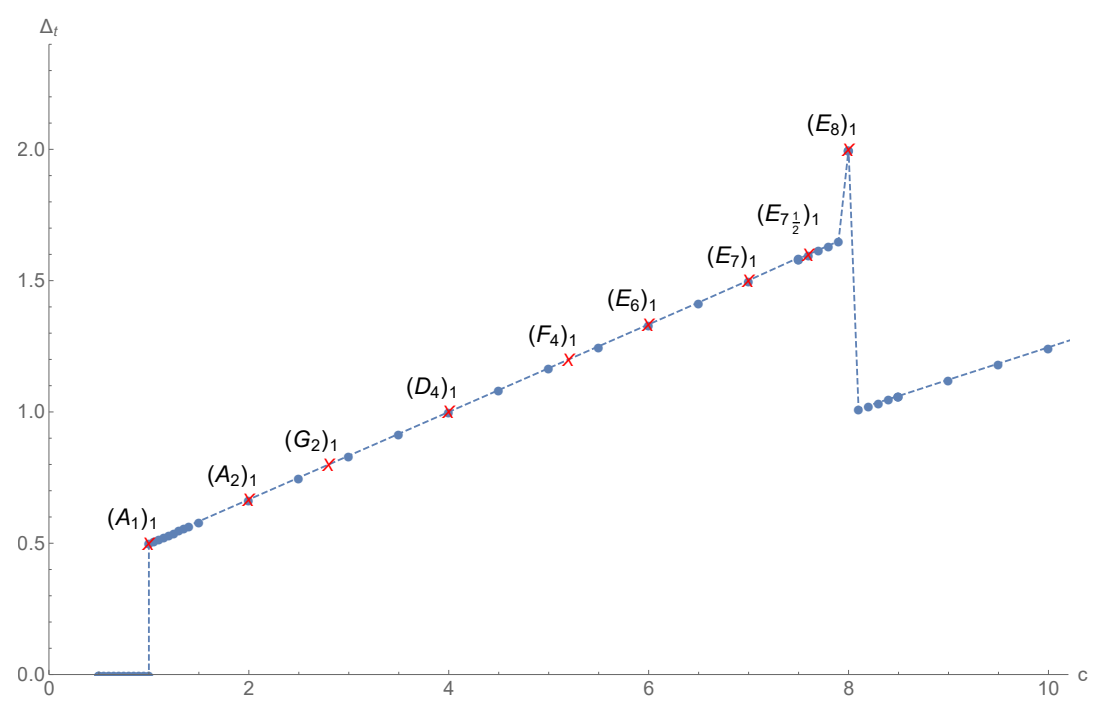

Figure 1. Numerical bound on the twist gap $\Delta_{t} \equiv \Delta-|j|$ for the non-current operators in the presence of holomorphic currents.

A similar bound in the presence of a U(1) global symmetry has been found in [27]. In [14], it was also observed that the level-one $\mathfrak{g}=A_{1}, A_{2}, G_{2}, D_{4}, E_{8}$ Wess-Zumino-Witten (WZW) models can be realized on the upper limit of the dimension gap of scalar primaries at the corresponding values of central charge. We further refine this analysis by either relaxing or imposing extra constraints on the spectrum. Let us highlight some of our results. We first assume the existence of the holomorphic currents, and investigate the bounds on the twist gap $\left(\Delta \geq \Delta_{t}+|j|\right)$ for the non-current primaries as a function of central charge $c$. The resulting numerical upper bound exhibits rather distinctive behavior compared to that of the upper bounds studied in [14]. It is natural to ask if any known theories are realized on the numerical boundary of the twist gap.

We find that the numerical boundary realizes the level-one WZW models with $\mathfrak{g}=A_{1}, A_{2}, G_{2}, D_{4}, F_{4}, E_{6}, E_{7}, E_{8}$, as illustrated in figure 1 . This set of $\mathfrak{g}$ agrees with the so-called Deligne's exceptional series. They are the simplest examples of rational conformal field theories (RCFT) with extended chiral algebras whose characters are given by the solutions of degree-two modular differential equation [28, 29] with

$$
c=1,2, \frac{14}{5}, 4, \frac{26}{5}, 6,7,8 \text {. }
$$

Moreover, we also find the modular invariant partition function for the $c=\frac{38}{5}$ realized at the numerical boundary. This partition function can be thought of as that of a two-channel RCFT with extended chiral algebra $\widehat{\mathfrak{g}}_{k=1}=\left(\widehat{E}_{7 \frac{1}{2}}\right)_{k=1}$, if such theory exists. ${ }^{2}$

Figure 2 shows the upper bound on the twist gap in the large central charge region. We find numerous kinks and peaks on the numerical boundary. Among them, the three points at $c=8, c=24$ and $c=48$ can be identified with the $\left(\widehat{E_{8}}\right)_{1}$ WZW, Monster CFT [30]

\footnotetext{
${ }^{2}$ The Lie algebra $E_{7 \frac{1}{2}}$ is a non-simple subalgebra of $E_{8}$, which shares common features like the ones in the Deligne exceptional series [47].
} 


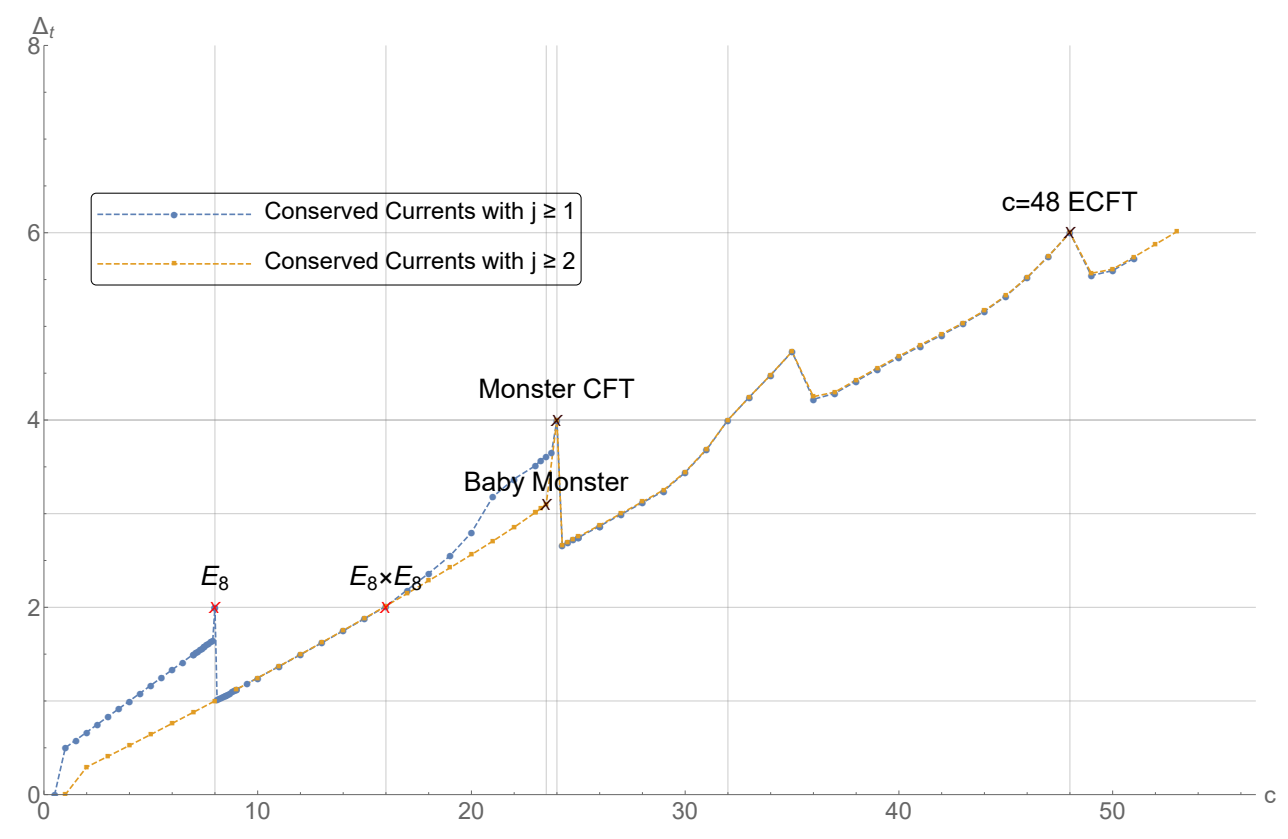

Figure 2. Numerical bound for the twist gap, in the presence of the conserved currents of spin $1 \leq j \leq j_{\max }$ and the conserved currents of $\operatorname{spin} 2 \leq j \leq j_{\max }$.

and $c=48$ extremal CFT, respectively. We also find that the $\left(\widehat{E_{8}}\right)_{1} \times\left(\widehat{E_{8}}\right)_{1} \mathrm{WZW}$ model can be placed at the boundary point at $c=16$. On the other hand, we obtain different numerical bounds on the twist gap depicted in figure 6 when the currents are restricted to have spins of $j \geq 2$. Interestingly, we are able to show that some RCFTs with finite group symmetry of large degree can sit on the numerical upper bound. For instance, the baby Monster CFT [31] is realized at the boundary point of $c=\frac{47}{2}$.

Finally, we impose the $\mathcal{W}$-algebra symmetry to our analysis, which can be applied to constraining higher-spin gravity in $\mathrm{AdS}_{3}$ [32]. This can be done by using the character for the $\mathcal{W}$-algebra instead of the Virasoro character in the equation (1.1). We assume that there is no degenerate state besides the vacuum. When the central charge is larger than the $\operatorname{rank} r=\operatorname{rank}(\mathfrak{g})$, the numerics do not show a significant difference with the Virasoro case. This might be due to the fact that we are not using the full character of the $\mathcal{W}$-algebra, but rather an 'unrefined' one for simplicity. However, we find that the numerical bound shows a sharp cliff at $c \sim r$, as presented in figure 3. It means there is no modular invariant partition function for $c<r$, which can be considered as a unitary bound of $\mathcal{W}_{k}$ algebra. Additionally, we find that the rank-three $\mathcal{W}\left(A_{3}\right)$ character realize the level-one $A_{3}$ WZW model at the numerical boundary, but not for the other choice of $\mathcal{W}(\mathfrak{g})$.

This paper is organized as follows. In section 2, we review the solutions to the degreetwo and degree-three modular differential equation (MDE). It is known that the solutions to the MDE can be identified with the vacuum and primary characters of a two-character or three-character RCFT. We also summarize the basic aspect of the 'unrefined' character of $\mathcal{W}$-algebra. In section 3 , we present numerical upper bounds on the scalar gap, overall gap and twist gap for the parity preserving CFTs with or without conserved currents. We 


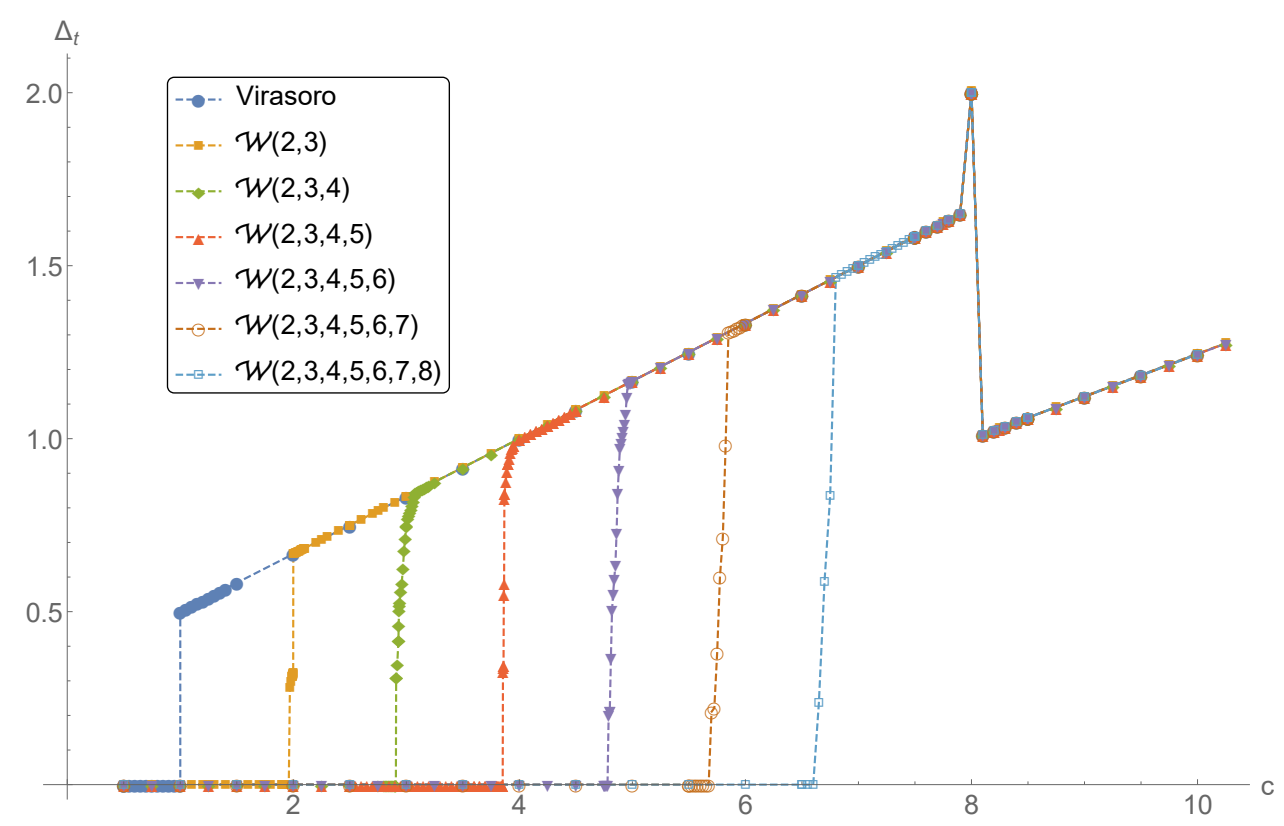

Figure 3. Numerical bounds on the twist gap with various $\mathcal{W}$-algebras. We also assume the presence of the conserved currents of $j \geq 1$ in the spectrum.

identify 16 RCFTs that are located on the numerical boundary. In section 4 , we provide the evidences for the identification of the 16 special points on the numerical boundary with the known and conjectured RCFTs. We find the modular invariant partition function of various RCFTs by assuming every extremal spectrum saturate the bounds on the maximal degeneracies. In section 5 , we repeat the numerical analysis with the character for the $\mathcal{W}$-algebra.

\section{Preliminaries}

\subsection{Modular differential equation}

A conformal field theory with a finite number of primary operators (maybe under an extended chiral algebra) is called a rational conformal field theory (RCFT). From this definition, the partition function of a RCFT can be expressed as

$$
Z(\tau, \bar{\tau})=\sum_{i, j=1}^{n} M_{i j} f_{i}(\tau) \bar{f}_{j}(\bar{\tau}),
$$

where $f_{i}(\tau)$ denote the characters of the (extended) chiral algebra including the Virasoro algebra.

There has been an attempt to classify RCFTs using the modular differential equation [28]. The main idea is to regard $n$ characters $f_{i}(\tau)$ of a given RCFT as independent solutions to a modular-invariant differential equation. One can use the covariant derivative on a modular form of weight $r$,

$$
\mathcal{D}_{\tau}=\partial_{\tau}-\frac{1}{6} i \pi r E_{2}(\tau)
$$


with the second Eisenstein series $E_{2}(\tau)$, to express the most general $n$-th order holomorphic differential equation as

$$
\left[\mathcal{D}_{\tau}^{n}+\sum_{k=0}^{n-1} \phi_{2(n-k)}(\tau) \mathcal{D}_{\tau}^{k}\right] f(\tau)=0
$$

Here $\phi_{2 k}(\tau)$ denotes a modular form of weight $2 k$ that becomes singular at the zeros of the Wrönskian $W=\operatorname{det} W_{j}^{i}$ with $W^{i}{ }_{j}=\mathcal{D}_{\tau}^{i} f_{j}(\tau)$ and $i, j=1, \ldots, n$. It is shown in [28] that the total number of zeroes of the Wrönskian $W$ is given by

$$
-\sum_{i=1}^{n-1} h_{i}+\frac{c}{24}+\frac{n(n-1)}{12}=\frac{\ell}{6}
$$

where $h_{i}$ denote the conformal weights of non-vacuum primary operators in a given RCFT and $\ell$ is either 0 or an integer greater than or equal to two.

In the present work, we are mostly interested in the modular differential equation with $\ell=0$ where $\phi_{2 k}(\tau)$ of $(2.3)$ can be expressed as a sum of monomials of $E_{4}(\tau)^{a} E_{6}(\tau)^{b}$ with $4 a+6 b=2 k$. The Eisenstein series are normalized such that they can be expanded as

$$
\begin{aligned}
& E_{4}(\tau)=1+240 q+2160 q^{2}+6720 q^{3}+17520 q^{4}+\mathcal{O}\left(q^{5}\right), \\
& E_{6}(\tau)=1-504 q-16632 q^{2}-122976 q^{3}-532728 q^{4}+\mathcal{O}\left(q^{5}\right)
\end{aligned}
$$

in the limit $q=e^{2 \pi i \tau} \rightarrow 0$. We further demand the modular differential equation to have a solution of the form

$$
f_{\mathrm{vac}}=q^{-\frac{c}{24}}\left[\prod_{m=2}^{\infty} \frac{1}{1-q^{m}}+\mathcal{O}\left(q^{n-1}\right)\right]
$$

which will be identified as the vacuum character of a corresponding RCFT with the central charge $c$. We can then determine the modular differential equations unambiguously up to fifth order. For instance, the modular differential equation of order two is given by

$$
\left[\left(q \frac{d}{d q}\right)^{2}-\frac{1}{6} E_{2}(\tau)\left(q \frac{d}{d q}\right)-\frac{c(c+4)}{576} E_{4}(\tau)\right] f(\tau)=0 .
$$

And the modular differential equation of order three is given by

$$
\left[\left(q \frac{d}{d q}\right)^{3}-\frac{1}{2} E_{2}(\tau)\left(q \frac{d}{d q}\right)^{2}+\left\{\frac{1}{24} E_{2}(\tau)^{2}-\alpha E_{4}(\tau)\right\}\left(q \frac{d}{d q}\right)+\beta E_{6}(\tau)\right] f(\tau)=0
$$

with

$$
\alpha=\frac{152+80 c+7 c^{2}}{5952}, \quad \beta=\frac{c\left(144+66 c+5 c^{2}\right)}{214272} .
$$




\subsection{Deligne's exceptional series and monsters}

Deligne's exceptional groups and WZW models. The "vacuum" character of (2.7) takes the following form

$$
f_{\mathrm{vac}}(\tau)=q^{-c / 24}\left[1+\frac{c(5 c+22)}{10-c} q+\cdots\right],
$$

which implies that the corresponding RCFT with central charge $c$ should contain $\frac{c(5 c+22)}{10-c}$ spin-one conserved currents. In other words, the chiral algebra of the RCFT we are looking for could be generated by Virasoro and also Kac-Moody algebras as long as $\frac{c(5 c+22)}{10-c}$ becomes a positive integer. When $c=-22 / 5$, there is no conserved-current. This gives the simplest (non-unitary) minimal model, the Yang-Lee model.

One can show that the Wess-Zumino-Witten (WZW) models for $A_{1}, A_{2}, G_{2}, D_{4}, F_{4}$, $E_{6}, E_{7}$ and $E_{8}$ with level one satisfy the above conditions. In general, the central charge of the $\widehat{\mathfrak{g}}_{k}$ WZW model with level $k$ is given by

$$
c\left(\widehat{\mathfrak{g}}_{k}\right)=\frac{k \operatorname{dim} \mathfrak{g}}{k+h^{\vee}},
$$

where $h^{\vee}$ denotes the dual Coxeter number of $\mathfrak{g}$. It is straightforward to show that

$$
\operatorname{dim} \mathfrak{g}=\frac{c\left(\widehat{\mathfrak{g}}_{1}\right)\left(5 c\left(\widehat{\mathfrak{g}}_{1}\right)+22\right)}{10-c\left(\widehat{\mathfrak{g}}_{1}\right)},
$$

when $\mathfrak{g}=A_{1}, A_{2}, G_{2}, D_{4}, F_{4}, E_{6}, E_{7}$ and $E_{8}$. These groups are often referred as the Deligne's exceptional series.

Indeed, $f_{\text {vac }}(\tau)$ of $(2.10)$ at $c=c\left(\widehat{\mathfrak{g}}_{1}\right)$ is the vacuum character of the WZW model for Deligne's exceptional groups with level one. Moreover, one can identify the other solution to $(2.7)$ with $c=c\left(\widehat{\mathfrak{g}}_{1}\right),^{3}$

$$
f_{h}(\tau)=a_{0} q^{h-\frac{c}{24}}\left[1+\frac{(5 c-2)(c+4)}{c+14} q+\cdots\right] \text { with } h=\frac{2+c}{12},
$$

as the characters associated with the primary operators of the corresponding theory; here $a_{0}$ is a constant which is not determined by the modular differential equation. The $\widehat{\mathfrak{g}}_{1} \mathrm{WZW}$ model with level one has primary operators in the dominant highest-weight representations of the affine algebra $\widehat{\mathfrak{g}}$, i.e.,

$$
k=1 \geq(\lambda, \theta)_{\widehat{\mathfrak{g}}},
$$

where $\theta$ is the highest root of the Lie algebra $\mathfrak{g}$ (see table 1 ). Their conformal weights are

$$
h_{\lambda}\left(\mathfrak{g}_{k}\right)=\frac{(\lambda, \lambda+2 \rho)}{2\left(k+h^{\vee}\right)},
$$

where $\rho$ denote the Weyl vector. The conformal weight $h_{\lambda}$ coincides with $\frac{1}{12}\left(2+c\left(\widehat{\mathfrak{g}}_{1}\right)\right)$ for the $\widehat{\mathfrak{g}}_{1}$ in the Deligne's exceptional series except for $E_{8}$. One can also show that,

\footnotetext{
${ }^{3}$ The exact solutions $f_{\mathrm{vac}}(\tau), f_{h}(\tau)$ of the second order MDE are given in [33] in terms of hypergeometric series.
} 


\begin{tabular}{|c|c|c|c|c|c|}
\hline $\mathfrak{g}$ & $h^{\vee}$ & $\operatorname{dim} \mathfrak{g}$ & $c\left(\widehat{\mathfrak{g}}_{1}\right)$ & dominant highest-weight rep. & $h_{\lambda}\left(\widehat{\mathfrak{g}}_{1}\right)$ \\
\hline$A_{1}$ & 2 & 3 & 1 & {$[0 ; 1]$} & $\frac{1}{4}$ \\
$A_{2}$ & 3 & 8 & 2 & {$[0 ; 1,0],[0 ; 0,1]$} & $\frac{1}{3}$ \\
$G_{2}$ & 4 & 14 & $\frac{14}{5}$ & {$[0 ; 1,0]$} & $\frac{2}{5}$ \\
$D_{4}$ & 6 & 28 & 4 & {$[0 ; 1,0,0,0],[0 ; 0,0,1,0],[0 ; 0,0,0,1]$} & $\frac{1}{2}$ \\
$F_{4}$ & 9 & 52 & $\frac{26}{5}$ & {$[0 ; 0,0,0,1]$} & $\frac{3}{5}$ \\
$E_{6}$ & 12 & 78 & 6 & {$[0 ; 1,0,0,0,0,0],[0 ; 0,0,0,0,1,0]$} & $\frac{2}{3}$ \\
$E_{7}$ & 18 & 133 & 7 & {$[0 ; 0,0,0,0,0,1,0]$} & $\frac{3}{4}$ \\
$E_{8}$ & 30 & 248 & 8 & none & \\
\hline
\end{tabular}

Table 1. Dual Coxeter numbers, dimensions, central charges $c\left(\mathfrak{g}_{k=1}\right)$, dominant affine weights of $\widehat{\mathfrak{g}}_{k=1}$ other than the basic weight $[1 ; 0,0, \ldots, 0]$, and conformal weights of corresponding primary operators.

when $a_{0}=\operatorname{dim} \lambda$, all the coefficients of the $q$-expansion of $f_{h}(\tau)(2.13)$ with $c=c\left(\widehat{\mathfrak{g}}_{1}\right)$ become positive integers that agree with those of the characters associated with the primary operators of weight $h=h_{\lambda}\left(\widehat{\mathfrak{g}}_{1}\right)$. For example, for $c=4$ (when we turn off the chemical potentials for the flavor charges),

$$
\begin{aligned}
f_{h=1 / 2}(\tau) & =\chi_{[0 ; 1,0,0,0]}(\tau)=\chi_{[0 ; 0,0,1,0]}(\tau)=\chi_{[0 ; 0,0,0,1]}(\tau) \\
& =8+64 q+288 q^{2}+1024 q^{3}+3152 q^{4}+8704 q^{5}+\cdots
\end{aligned}
$$

where $\chi_{[0 ; \lambda]}$ are the characters associated with the primary operators with the highestweights $\lambda$ that are present in the $\widehat{s o}(8)_{1} \mathrm{WZW}$ model. The three representations are mapped to each other via triality of $s o(8)$.

For later convenience, let us also discuss the modular-invariant partition function of the WZW models with level one. For the Deligne exceptional groups, it is shown in [34] that the torus partition functions (in the limit of zero chemical potential for the global currents) are

$$
Z_{\widehat{\mathfrak{g}}_{1}}(\tau, \bar{\tau})=f_{\mathrm{vac}}(\tau) \bar{f}_{\mathrm{vac}}(\bar{\tau})+N\left(\widehat{\mathfrak{g}}_{1}\right) f_{h}(\tau) \bar{f}_{h}(\bar{\tau}),
$$

where $N\left(\widehat{\mathfrak{g}}_{1}\right)$ denotes the number of dominant highest-weight representations of $\widehat{\mathfrak{g}}_{1}$ other than the basic representation. For instance, the partition function of $\widehat{s o}(8)_{1}$ WZW model can be expressed as

$$
Z_{\widehat{s o}(8)_{1}}(\tau, \bar{\tau})=f_{\text {vac }}(\tau) \bar{f}_{\mathrm{vac}}(\bar{\tau})+3 f_{h=1 / 2}(\tau) \bar{f}_{\bar{h}=1 / 2}(\bar{\tau})
$$

with $c=4$.

Monster and its cousins. The modular differential equation of order three (2.8) with the ansatz (2.6) has been studied relatively recently in order to explore unitary RCFTs that has no Kac-Moody symmetry (but may have some extended chiral algebras) [35, 36]. 
Let us first discuss the possible values of the central charge for which RCFTs without the Kac-Moody symmetry may exist. The "vacuum character" of (2.8) is given by

$$
\begin{aligned}
f_{\mathrm{vac}}(\tau)= & q^{-\frac{c}{24}}\left[1+\frac{c\left(70 c^{2}+955 c+2388\right)}{2((c-55) c+748)} q^{2}\right. \\
& \left.+\frac{5530 c^{5}+114000 c^{4}+919648 c^{3}+3949824 c^{2}+5656576 c}{3\left(c^{2}-86 c+1864\right)\left(c^{2}-55 c+748\right)} q^{3}+\cdots\right] .
\end{aligned}
$$

It is not difficult to show that the requirement to have positive integer coefficients of the $q$-expansion of $f_{\text {vac }}(\tau)$ (up to the $\mathcal{O}\left(q^{400}\right)$ ) restrict the central charge $c$ to nine possible values $[35,36]$,

$$
c=-\frac{44}{5}, 8,16, \frac{47}{2}, 24,32, \frac{164}{5}, \frac{236}{7}, 40 .
$$

One can also identify the other two solutions to (2.8) with two different characters associated with the primary operators of conformal weights $h=h_{ \pm}(c)$,

$$
f_{h_{ \pm}}(\tau)=q^{h_{ \pm}-\frac{c}{24}}\left[a_{0}+\mathcal{O}(q)\right]
$$

with

$$
h_{ \pm}(c)=\frac{c+4}{16} \pm \frac{\sqrt{(24-c) c+368}}{16 \sqrt{31}}
$$

in the corresponding would-be RCFT with central charge $c$. It is clear from (2.22) that the above two "primary operators" of weights $h_{ \pm}(c)$ would not exist for $c \geq 12+16 \sqrt{2}$. This suggests that the RCFT of our interest with $c=40$ may correspond to a single-character RCFT, having the vacuum as the only primary.

The vacuum character of a single-character theory has to be of the form [28]

$$
j(\tau)^{\alpha}(j(\tau)-1728)^{\beta} P_{k}(j(\tau)),
$$

where $\alpha=0,1 / 3,2 / 3$ and $\beta=0,1 / 2$ while $P_{k}(j(\tau))$ denotes a polynomial of order $k$ in the $j$-function with integer coefficients. ${ }^{4}$ One can indeed show that $f_{\text {vac }}(\tau)(2.19)$ for $c=40$ can be expressed in terms of the $j$-function,

$$
\begin{aligned}
f_{\text {vac }}(\tau) & =q^{-\frac{40}{24}}\left[1+20620 q^{2}+86666240 q^{3}+\cdots\right] \\
& =j^{\frac{2}{3}}(\tau)[j(\tau)-1240] .
\end{aligned}
$$

In summary, it is plausible from (2.8) with (2.6) that unitary RCFTs without KacMoody algebra can exist for $c=8,16,47 / 2,24,32,164 / 5,236 / 7$ and 40. (See table 2.) Some

${ }^{4}$ Here the $j$-function is normalized as follows:

$$
j(\tau)=\frac{\left(12 E_{4}(\tau)\right)^{3}}{E_{4}(\tau)^{3}-E_{6}(\tau)^{2}} .
$$




\begin{tabular}{|c|c|c|c|}
\hline$c$ & $f_{\mathrm{vac}}(\tau)$ & $f_{h_{ \pm}}(\tau)$ & automorphism group \\
\hline 8 & $q^{-\frac{8}{24}}\left[1+156 q^{2}+\cdots\right]$ & $\left\{\begin{array}{l}q^{\frac{1}{2}-\frac{8}{24}} a_{0}[1+36 q+\cdots] \\
q^{1-\frac{8}{24}} b_{0}[1+16 q+\cdots]\end{array}\right.$ & $O_{10}^{+}(2) .2$ \\
\hline 16 & $q^{-\frac{16}{24}}\left[1+2296 q^{2}+\cdots\right]$ & $\left\{\begin{array}{l}q^{1-\frac{16}{24}} a_{0}[1+136 q+\cdots] \\
q^{\frac{3}{2}-\frac{16}{24}} b_{0}[1+52 q+\cdots]\end{array}\right.$ & $O_{10}^{+}(2) .2^{16}$ \\
\hline$\frac{47}{2}$ & $q^{-\frac{47}{48}}\left[1+96256 q^{2}+\cdots\right]$ & $\left\{\begin{array}{l}q^{\frac{3}{2}-\frac{47}{48}} a_{0}[3+785 q+\cdots] \\
q^{\frac{31}{16}-\frac{47}{48}} b_{0}[47+5177 q+\cdots]\end{array}\right.$ & Baby Monster \\
\hline 24 & $q^{-\frac{24}{24}}\left[1+196884 q^{2}+\cdots\right]$ & none & Monster \\
\hline 32 & $q^{-\frac{32}{24}}\left[1+139504 q^{2}+\cdots\right]$ & none & unknown \\
\hline$\frac{164}{5}$ & $q^{-\frac{41}{30}}\left[1+90118 q^{2}+\cdots\right]$ & $\left\{\begin{array}{l}q^{\frac{11}{5}-\frac{41}{30}}[248+90365 q+\cdots] \\
q^{\frac{12}{5}-\frac{41}{30}}[484+120032 q+\cdots]\end{array}\right.$ & unknown \\
\hline$\frac{236}{7}$ & $q^{-\frac{59}{42}}\left[1+63366 q^{2}+\cdots\right]$ & $\left\{\begin{array}{l}q^{\frac{16}{7}-\frac{59}{42}}[391+140896 q+\cdots] \\
q^{\frac{17}{7}-\frac{59}{42}}[7192+1971507 q+\cdots]\end{array}\right.$ & unknown \\
\hline 40 & $q^{-\frac{40}{24}}\left[1+20620 q^{2}+\cdots\right]$ & none & unknown \\
\hline
\end{tabular}

Table 2. Central charge, characters with unfixed integers $a_{0}$ and $b_{0}$, and automorphism group of eight possible rational conformal field theories.

of their vacuum characters (2.19) can be identified with the Monster module $(c=24)$ [37] and the Höhn Baby Monster module $(c=47 / 2)$ [31]. Furthermore, the vacuum characters for $c=8,16$ can be realized as a certain fixed-point free lattice for the rank 8 even lattice and the rank 16 Barnes-Wall even lattice whose automorphism groups are related to $O_{10}^{+}(2) .2$ and $O_{10}^{+}(2) .2^{16}$ respectively. The extended chiral algebras for $c=32, \frac{164}{5}, \frac{236}{7}, 40$ are however not much known and need further investigation. Moreover, beyond the Monster CFT, some of their modular-invariant partition functions have been poorly understood.

We discuss in the next section that the above RCFTs, both of the WZW models with level one for the Deligne's exceptional series and the Monster CFT and its cousins, are realized at the numerical bounds of the twist gap.

\subsection{Character of $\mathcal{W}$-algebra}

$\mathcal{W}$-algebra is an extension of the Virasoro algebra, augmented with generators with higher spins $s \geq 2$. In section 5 , we will investigate consequences of the $\mathcal{W}$-algebra symmetry in the modular invariant partition function. We label the $\mathcal{W}$-algebras by the spins (or dimensions) of the generators as $\mathcal{W}\left(d_{1}, d_{2}, \ldots, d_{r}\right)$, where we call $r$ as the rank of the algebra. For the $\mathcal{W}(\mathfrak{g})$-algebra associated to a Lie algebra $\mathfrak{g}$, the spins of the generators agree with the degrees of the Casmirs of $\mathfrak{g}$. In this section, we briefly review necessary aspects of the $\mathcal{W}$-algebra for our numerical bootstrap program. See [38] for more details.

$\mathcal{W}(\mathbf{2}, \mathbf{3})$-algebra. The first example of $\mathcal{W}$ algebra is the $\mathcal{W}\left(A_{2}\right)=\mathcal{W}(2,3)$ [39]. It has two generators, one comes from the stress tensor $T(z)$, and the spin-3 generator $W(z)$, 
which upon mode expansion

$$
T(z)=\sum_{n \in \mathbb{Z}} L_{n} z^{-n-2}, \quad W(z)=\sum_{n \in \mathbb{Z}} W_{n} z^{-n-3} .
$$

The commutation relations between generators are given by

$$
\begin{aligned}
{\left[L_{m}, L_{n}\right]=} & (m-n) L_{m+n}+\frac{c}{12} m\left(m^{2}-1\right) \delta_{m+n, 0} \\
{\left[L_{m}, W_{n}\right]=} & (2 m-n) W_{m+n} \\
{\left[W_{m}, W_{n}\right]=} & (m-n)\left[\frac{1}{15}(m+n+3)(m+n+2)-\frac{1}{6}(m+2)(n+2)\right] L_{m+n} \\
& +\frac{16}{22+5 c}(m-n) \Lambda_{m+n}+\frac{c}{360} m\left(m^{2}-1\right)\left(m^{2}-4\right) \delta_{m+n, 0}
\end{aligned}
$$

where the operator $\Lambda_{n}$ is

$$
\Lambda_{n}=\sum_{p \leq-2} L_{p} L_{n-p}+\sum_{p \geq-1} L_{n-p} L_{p}-\frac{3}{10}(n+2)(n+3) L_{n} .
$$

The highest weight states $|h, w ; c\rangle$ are labeled by $L_{0}$ eigenvalue $h$ and $\mathcal{W}_{0}$ eigenvalue $w$ and also by the central charge $c$. The Verma module is generated by acting negative modes $L_{-m}$ and $\mathcal{W}_{-m}$ to the highest weight state. When the central charge $c$ and the highest weights $(h, w)$ satisfy some relation, the Verma module might carry null states that we need to mod out to form a faithful representation. For a generic value of $c, h, w$, the Verma module does not have a null state, which is the scenario we are mostly interested in.

One can define the character for a given representation $\mathcal{V}_{h, w}^{c}$ as

$$
\chi_{(h, w ; c)}(\tau ; p)=\operatorname{Tr}_{\mathcal{V}_{h, w}^{c}}\left(q^{L_{0}-\frac{c}{24}} p^{W_{0}}\right) .
$$

This quantity turns out to be rather challenging to compute, even for the case of the Verma module. This has to do with the fact that one needs to simultaneously diagonalize vectors in $\mathcal{V}_{h, w}$ with respect to $L_{0}$ and $W_{0}$. See $[40,41]$ for a recent development on this issue. Due to its computational difficulty, we will focus on the 'unrefined' character, which sets $p=1$. The unrefined character turns out to be very simple, just given by

$$
\chi_{(h, w ; c)}(\tau)=\operatorname{Tr}_{\mathcal{V}_{h, w}^{c}} q^{L_{0}-\frac{c}{24}}=q^{h-\frac{c}{24}} \prod_{n \geq 1} \frac{1}{\left(1-q^{n}\right)^{2}}=\frac{q^{h-\frac{c-2}{24}}}{\eta(\tau)^{2}},
$$

for a generic representation. For the vacuum module, we simply get

$$
\chi_{0}(\tau)=q^{-\frac{c}{24}} \prod_{n=2}^{\infty} \frac{1}{\left(1-q^{n}\right)} \prod_{n=3}^{\infty} \frac{1}{\left(1-q^{n}\right)}=\frac{q^{-\frac{c-2}{24}}(1-q)^{2}\left(1-q^{2}\right)}{\eta(\tau)^{2}}
$$

from the following null states:

$$
\left\langle 0\left|L_{1} L_{-1}\right| 0\right\rangle=0, \quad\left\langle 0\left|W_{1} W_{-1}\right| 0\right\rangle=0, \quad\left\langle 0\left|W_{2} W_{-2}\right| 0\right\rangle=0 .
$$


$\mathcal{W}$-algebra associated to a Lie algebra. The most straightforward way of constructing a $\mathcal{W}$-algebra is to start with generators of dimensions $d_{1}, \ldots d_{r}$ and then try to fix various structure constants by imposing Jacobi identity. This way is notoriously difficult to perform in practice, which has been done only up to 3 generators [42]. Instead, a more systematic approach is available.

Start with an affine Kac-Moody algebra $\widehat{\mathfrak{g}}_{k}$. From here, one can obtain an associated $\mathcal{W}$-algebra via quantum Drinfeld-Sokolov reduction [43-45]. This $\mathcal{W}$-algebra $\mathcal{W}\left(\widehat{\mathfrak{g}}_{k}, \Lambda\right)$ is labeled by choice of a $\mathfrak{s u}(2)$ embedding $\Lambda: \mathfrak{s u}(2) \hookrightarrow \mathfrak{g}$, where $\mathfrak{g}$ is the finite part of the affine Kac-Moody algebra. When $\mathfrak{g}=\mathfrak{s u}(N)$, the $\mathfrak{s u}(2)$ embedding is classified in terms of partitions of $N$ or Young tableaux of $N$ boxes. This choice determines how the Virasoro algebra is realized in affine Kac-Moody algebra.

The choice of $\Lambda$ determines the degrees of the $\mathcal{W}$-algebra generators. It goes as follows. One can decompose the adjoint representation of $\mathfrak{g}$ into the $\mathfrak{s u}(2)$ representations as

$$
\operatorname{adj}(\mathfrak{g})=\bigoplus_{j} V_{j}
$$

where $V_{j}$ denotes $2 j+1$ dimensional spin- $j$ representation under $\mathfrak{s u}(2)$. Once we have the decomposition as above, the $\mathcal{W}(\mathfrak{g}, \Lambda)$-algebra associated to the affine Lie algebra $\widehat{\mathfrak{g}}$ and the $\mathfrak{s u}(2)$ embedding $\Lambda$ will have the generators of spins (dimensions) given by $j+1$. For example, let us choose $\Lambda$ to be given by the principal embedding $\Lambda_{\mathrm{pr}}$. Then the adjoint representation decomposes into $\mathfrak{g}=\oplus_{i=1}^{r} V_{d_{i}-1}$ where $r$ is the rank of $\mathfrak{g}$ and $d_{i}$ are given by the degrees of the Casimir operators. Sometimes we denote the $\mathcal{W}$-algebra given by the principal embedding as $\mathcal{W}(\mathfrak{g}) \equiv \mathcal{W}\left(\mathfrak{g}, \Lambda_{\text {pr }}\right)$. Our main focus in section 5 will be the modular constraint coming from $\mathcal{W}(\mathfrak{g})$-algebra.

For the $\mathcal{W}\left(d_{1}, \ldots, d_{r}\right)$-algebra, we have generators with the mode expansions given as

$$
W^{\left(d_{i}\right)}(z)=\sum_{n \in \mathbb{Z}} W_{n}^{\left(d_{i}\right)} z^{-n-d_{i}}
$$

Verma module is simply generated by acting negative modes of the generators $W_{-n}^{\left(d_{i}\right)}$ on the highest-weight state. Therefore, the reduced character for a generic module (that does not have any null state) is simply given as

$$
\chi_{h}(\tau)=q^{h-\frac{c}{24}} \prod_{n \geq 1} \frac{1}{\left(1-q^{n}\right)^{r}}=\frac{q^{h-\frac{c-r}{24}}}{\eta(\tau)^{r}},
$$

where we omitted the dependence on the weights for the generators except for the stressenergy tensor. The vacuum states are defined to be annihilated by all the generators of mode number greater than equal to zero. In addition, there are null states at level $1,2, \ldots, d-1$ of the form $W_{-n_{1}}^{(d)} \ldots W_{-n_{k}}^{(d)}|0\rangle$ with $1 \leq \sum_{k} n_{k} \leq d-1$. Now, we can write the vacuum character for general rank- $r \mathcal{W}\left(d_{1}, d_{2}, \ldots, d_{r}\right)$-algebra as

$$
\chi_{0}(\tau)=q^{-\frac{c}{24}} \prod_{i=1}^{r} \prod_{n=d_{i}}^{\infty} \frac{1}{\left(1-q^{n}\right)}=\frac{q^{-\frac{c-r}{24}}}{\eta(\tau)^{r}} \prod_{i=1}^{r} \prod_{j=1}^{d_{i}-1}\left(1-q^{j}\right) .
$$


Now, the partition function of a CFT with $\mathcal{W}\left(d_{1}, \ldots, d_{r}\right)$-algebra symmetry should be written in terms of $\mathcal{W}$-algebra characters, instead of Virasoro characters. For the computational convenience, we mainly focus on the reduced partition function given as

$$
\widehat{Z}(\tau, \bar{\tau})=|\tau|^{\frac{r}{2}}|\eta(\tau)|^{2 r} Z(\tau, \bar{\tau})
$$

Note that $|\tau|^{\frac{1}{2}}|\eta(\tau)|^{2}$ is modular invariant. Therefore the reduced partition function is also invariant under $\tau \rightarrow-\frac{1}{\tau}$.

\section{Modular constraint with Virasoro algebra}

\subsection{The modular bootstrap equation}

The torus partition function of a two-dimensional compact (bosonic) CFT can be defined as

$$
Z(\tau, \bar{\tau})=\operatorname{Tr}_{\mathcal{H}\left(S^{1}\right)}\left[q^{L_{0}-\frac{c_{L}}{24}} \bar{q}^{\bar{L}_{0}-\frac{c_{R}}{24}}\right] \text { with } q=e^{2 \pi i \tau},
$$

where $\tau$ parametrizes the complex structure of the torus, and trace is taken over the states of a given CFT on a unit circle. We focus on CFTs having parity-invariant spectrum and thus free from the gravitational anomaly, i.e., $c_{L}=c_{R}$ in what follows.

One can decompose the partition function $Z(\tau, \bar{\tau})$ of a given parity-preserving CFT in terms of the Virasoro characters as

$$
\begin{aligned}
Z(\tau, \bar{\tau})= & \chi_{0}(\tau) \bar{\chi}_{0}(\bar{\tau})+\sum_{h, \bar{h}} d_{h, \bar{h}}\left[\chi_{h}(\tau) \bar{\chi}_{\bar{h}}(\bar{\tau})+\chi_{\bar{h}}(\tau) \bar{\chi}_{h}(\bar{\tau})\right] \\
& +\sum_{j=1} d_{j}\left[\chi_{j}(\tau) \bar{\chi}_{0}(\bar{\tau})+\chi_{0}(\tau) \bar{\chi}_{j}(\bar{\tau})\right]
\end{aligned}
$$

where $\chi_{h}(\tau)$ denotes the Virasoro character for the highest-weight representation with weight $h$. The vacuum and non-vacuum characters take the forms

$$
\begin{gathered}
\chi_{0}(\tau)=q^{-\frac{c}{24}} \prod_{n=2}^{\infty} \frac{1}{1-q^{n}}, \\
\chi_{h>0}(\tau)=q^{h-\frac{c}{24}} \prod_{n=1}^{\infty} \frac{1}{1-q^{n}} .
\end{gathered}
$$

Unless a given CFT suffers from the large diffeomorphism anomaly, the torus partition function (3.1) has to be invariant under the modular transformation $\operatorname{SL}(2, \mathbb{Z})$ generated by $T$ and $S$,

$$
T: \tau \rightarrow \tau+1, \quad S: \tau \rightarrow-\frac{1}{\tau} .
$$

Invariance of the partition function under the $T$-transformation requires that all states carry integer spins, i.e., $j=|h-\bar{h}| \in \mathbb{Z}_{\geq 0}$. The invariance under the $S$-transformation implies that the spectrum $d_{h, \bar{h}}$ and $d_{j}$ are further constrained to satisfy

$$
0=\mathcal{Z}_{\mathrm{vac}}(\tau, \bar{\tau})+\sum_{h, \bar{h}} d_{h, \bar{h}}\left[\mathcal{Z}_{h, \bar{h}}(\tau, \bar{\tau})+\overline{\mathcal{Z}}_{h, \bar{h}}(\tau, \bar{\tau})\right]+\sum_{j=1} d_{j}\left[\mathcal{Z}_{j}(\tau, \bar{\tau})+\overline{\mathcal{Z}}_{j}(\tau, \bar{\tau})\right]
$$


where

$$
\begin{aligned}
\mathcal{Z}_{\mathrm{vac}}(\tau, \bar{\tau}) & =\chi_{0}(\tau) \bar{\chi}_{0}(\bar{\tau})-\chi_{0}\left(-\frac{1}{\tau}\right) \bar{\chi}_{0}\left(-\frac{1}{\bar{\tau}}\right), \\
\mathcal{Z}_{h, \bar{h}}(\tau, \bar{\tau}) & =\chi_{h}(\tau) \bar{\chi}_{\bar{h}}(\bar{\tau})-\chi_{h}\left(-\frac{1}{\tau}\right) \bar{\chi}_{\bar{h}}\left(-\frac{1}{\bar{\tau}}\right), \\
\mathcal{Z}_{j}(\tau, \bar{\tau}) & =\chi_{j}(\tau) \bar{\chi}_{0}(\bar{\tau})-\chi_{j}\left(-\frac{1}{\tau}\right) \bar{\chi}_{0}\left(-\frac{1}{\bar{\tau}}\right) .
\end{aligned}
$$

Our goal in this paper is to study the consequences of the equation (3.5) on the spectrum of operators. It is in general challenging to solve the constraint equation (3.5) analytically. However, the numerical method of semi-definite programming (SDP) makes it feasible to study the constraint imposed by (3.5). The procedure goes as follows: first, make a hypothesis on the CFT spectrum. Second, search for a linear functional $\alpha$ satisfying the conditions below

$$
\alpha\left[\mathcal{Z}_{\mathrm{vac}}(\tau, \bar{\tau})\right]=1, \quad \alpha\left[\mathcal{Z}_{h, \bar{h}}(\tau, \bar{\tau})\right] \geq 0, \quad \alpha\left[\mathcal{Z}_{j}(\tau, \bar{\tau})\right] \geq 0
$$

for $(h, \bar{h})$ subject to the hypothesis. If such an $\alpha$ exists, the non-negativity of $d_{h, \bar{h}}$ and $d_{j}$ implies that

$$
\alpha\left[\mathcal{Z}_{\mathrm{vac}}(\tau, \bar{\tau})\right]+\sum_{h, \bar{h}} d_{h, \bar{h}} \alpha\left[\mathcal{Z}_{h, \bar{h}}(\tau, \bar{\tau})+\overline{\mathcal{Z}}_{h, \bar{h}}(\tau, \bar{\tau})\right]+\sum_{j=1} d_{j} \alpha\left[\mathcal{Z}_{j}(\tau, \bar{\tau})+\overline{\mathcal{Z}}_{j}(\tau, \bar{\tau})\right]>0
$$

We thus find a contradiction that (3.5) cannot be satisfied, and the hypothetical CFT spectrum is ruled out.

In this section, we investigate the numerical upper bounds on the so-called scalar gap, overall gap and twist gap defined below for parity-preserving CFTs with and without conserved currents:

1. scalar gap problem: we impose a gap $\Delta_{s}$ in the spectrum of scalar primaries. In other words, the spectrum of a hypothetical CFT is constrained to have scalar primaries with conformal dimensions $\Delta \geq \Delta_{s}$ while the unitary bound $\Delta \geq j$ is satisfied for other primaries with spin $j$.

2. overall gap problem: there is a gap $\max \left(\Delta_{o}, j\right)$ in the conformal dimensions of all non-degenerate primaries. The conformal dimension $\Delta$ of non-degenerate primaries of spin $j$ are required to satisfy $\Delta \geq \max \left(\Delta_{o}, j\right)$. However, we do not impose the above gap on the conserved currents. If $\Delta_{o} \leq 1$, this condition is identical to the scalar gap problem. As the maximal gap $\Delta_{o}$ grows, one can expect to have upper bounds on $\Delta_{o}$ different to those of the scalar gap problem.

3. twist gap problem: we also study the universal gap $\Delta_{t}$ on the twist $t$ defined by $t=\Delta-j=2 \min (h, \bar{h})$. The gap is again relaxed for the conserved currents. A putative CFT is thus constrained to have non-degenerate primaries with conformal dimensions $\Delta \geq j+\Delta_{t}$ only. Among the three problems, the twist gap problem is expected to show the most stringent upper bound. 
It is convenient to use a linear functional $\alpha$ of the form

$$
\alpha=\left.\sum_{p=0}^{N} \sum_{m+n=2 p+1}\left(\tau \frac{\partial}{\partial \tau}\right)^{m}\left(\bar{\tau} \frac{\partial}{\partial \bar{\tau}}\right)^{n}\right|_{\tau=i, \bar{\tau}=-i}
$$

to implement the semi-definite programming problem (3.7). The spins of primaries are in practice truncated up to $j \leq j_{\max }$ where $j_{\max }$ is carefully chosen such that the numerical bounds for $\Delta_{s}, \Delta_{o}$ and $\Delta_{t}$ are well-stabilized for a given derivative order $N_{\max }=2 N+1$. In the present work, the default value for the derivative order is 41 , but can occasionally be enhanced up to 81 if necessary. In order to make the numerical analysis simple, we solve a different but equivalent SDP problem (3.7) with the partition function and characters multiplied by certain modular-invariant factors to get

$$
\begin{aligned}
\widehat{Z}(\tau, \bar{t}) & =\left|\tau^{\frac{1}{4}} \eta(\tau)\right|^{2} Z(\tau, \bar{\tau}), \\
\widehat{\chi}(\tau) & =\tau^{\frac{1}{4}} \eta(\tau) \chi(\tau) .
\end{aligned}
$$

Here $\widehat{Z}(\tau, \bar{\tau})$ and $\widehat{\chi}(\tau)$ are often referred to as the reduced partition function and reduced characters respectively $[12,14]$.

\subsection{Numerical bounds on spectrum gap}

We solve the SDP problems (3.7) with the scalar, overall and twist gaps using the SDPB package [26]. Figure 4 shows the numerical upper bounds on $\Delta_{s}, \Delta_{o}$ and $\Delta_{t}$ for paritypreserving CFTs with and without conserved currents.

As studied in [14], there is no essential difference between the CFTs with and without conserved currents for the numerical bounds on the scalar gap $\Delta_{s}$, especially when $c \leq 8$. The authors of [14] found that $\left(\widehat{A}_{1}\right)_{1},\left(\widehat{A}_{2}\right)_{1},\left(\widehat{G}_{2}\right)_{1},\left(\widehat{D}_{4}\right)_{1}$ and $\left(\widehat{E}_{8}\right)_{1}$ WZW models are realized on the numerical bounds at $c=1,2, \frac{14}{5}, 4,8$. It is rather tempting to test the possibility of achieving all the WZW models with Deligne's exceptional series $A_{1} \subset A_{2} \subset G_{2} \subset$ $D_{4} \subset F_{4} \subset E_{6} \subset E_{7} \subset E_{8}$ at the numerical boundary. Figure 5, however, shows that other level one WZW models for the Deligne's exceptional series would not be realized on the boundary even for the sufficiently large order of derivatives. It was also reported in [14] that there is no upper bound on the scalar gap $\Delta_{s}$ beyond $c \geq 25$ where one can easily construct a modular invariant "partition function" of a noncompact CFT having no scalar primaries.

Unlike the scalar gap $\Delta_{s}$, we find that the numerical bounds of the overall gap $\Delta_{o}$ are sensitive to the existence of conserved currents. Note that we do not impose the gap condition on the conserved currents. It turns out that the numerical bound on $\Delta_{o}$ for the CFT with conserved currents start to deviate from that of the CFT without conserved currents at $c=4$. We find a sharp peak at $c=8$ when the conserved currents are included, which can be identified again with the $\left(\widehat{E}_{8}\right)_{1}$ WZW model.

We observe that the curve for the numerical bounds on the twist gap $\Delta_{t}$ with the conserved currents shows the most dramatic pattern. We find that all the level one WZW models for the Deligne's exceptional series including $\left(\widehat{F}_{4}\right)_{1},\left(\widehat{E}_{6}\right)_{1}$ and $\left(\widehat{E}_{7}\right)_{1}$ now sit on the numerical bounds. We also observe that a modular invariant partition function with 


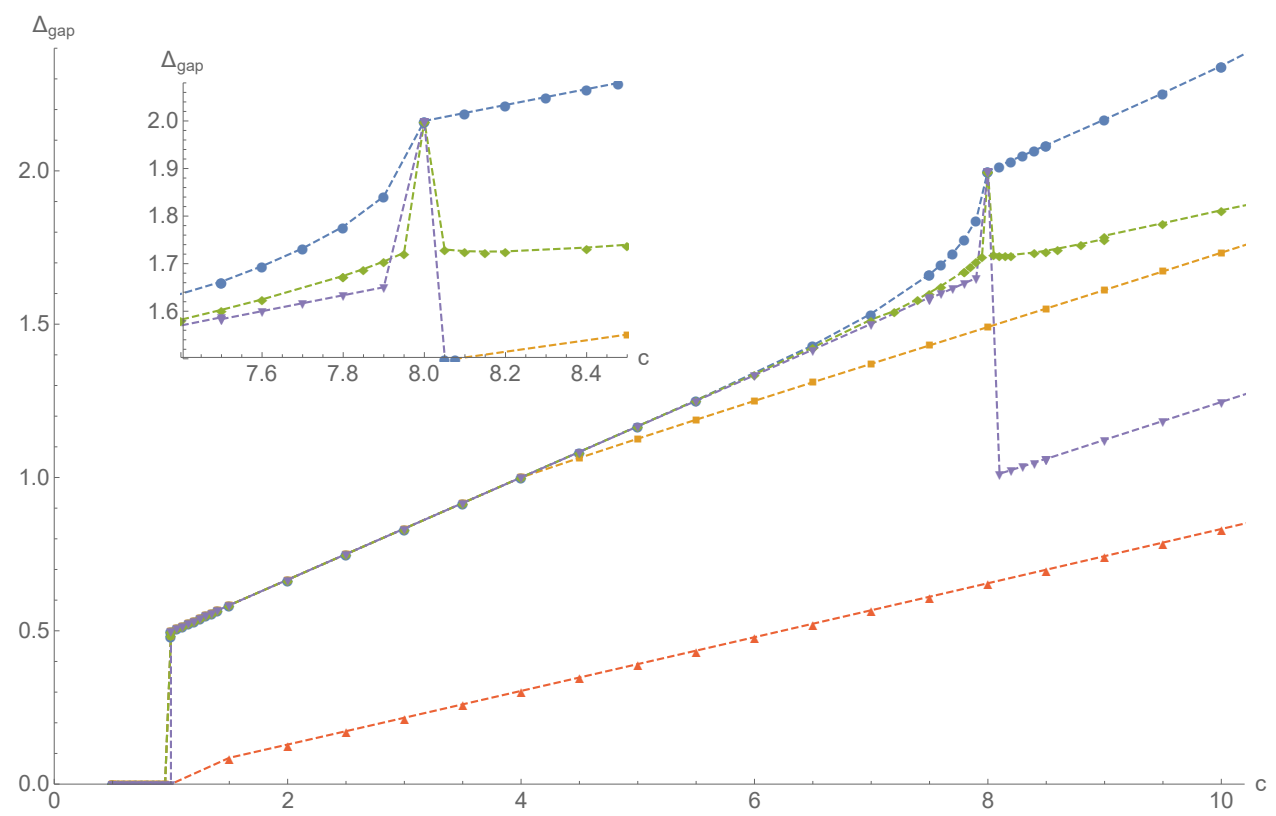

(a) Bounds in the region $1 \leq c \leq 10$. We zoom near the region around $c=8$.

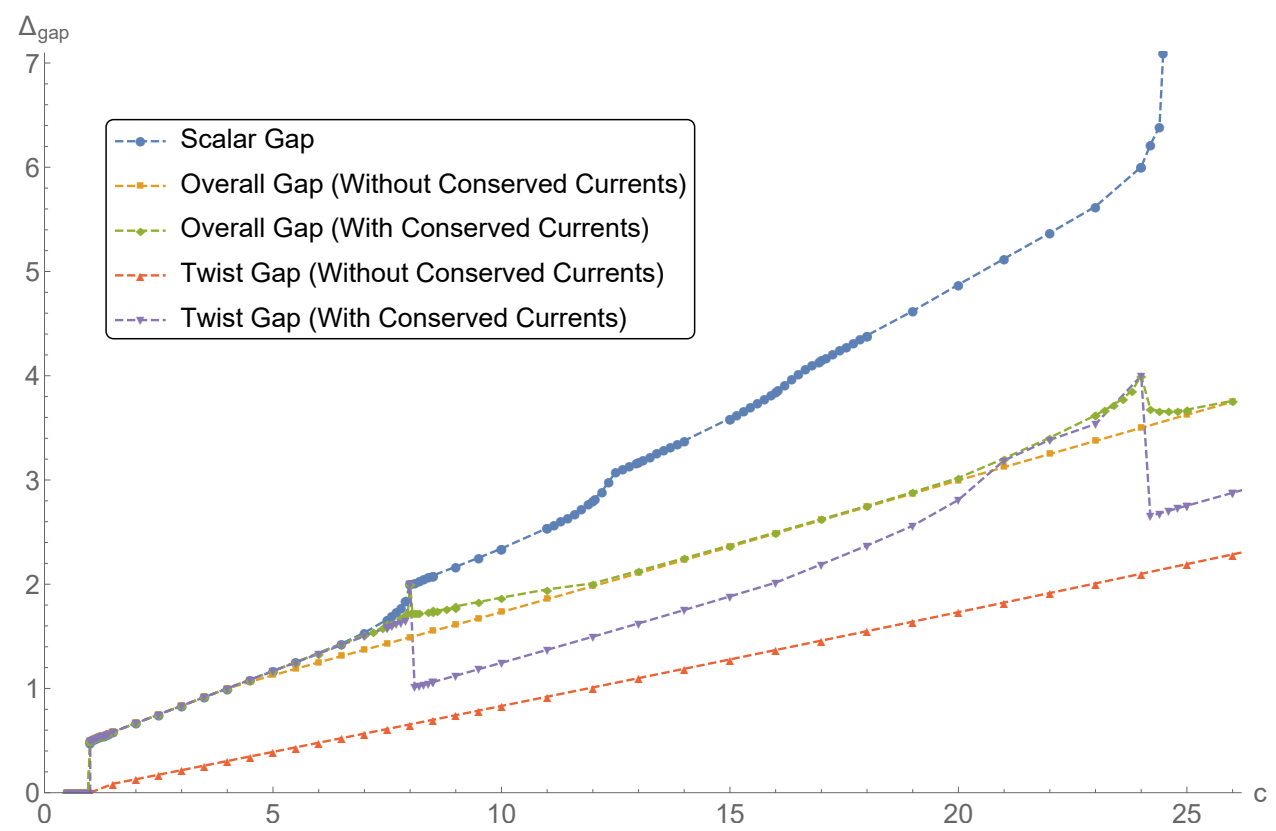

(b) Bounds in the region $1 \leq c \leq 26$.

Figure 4. Numerical upper bounds on the scalar gap $\Delta_{s}$, the overall gap $\Delta_{o}$, and the twist gap $\Delta_{t}$.

integer degeneracies is realized at the bound for $c=\frac{38}{5}$. Interestingly, this value of central charge is identical to the Sugawara central charge for $\widehat{E}_{7 \frac{1}{2}}$ with level 1 . We discuss this point in more detail in section 4.2.

The twist gap problem without the conserved currents also shows a different numerical upper bound. The asymptotic slope of this bound is compatible with $\frac{c-1}{12}[13]$. 


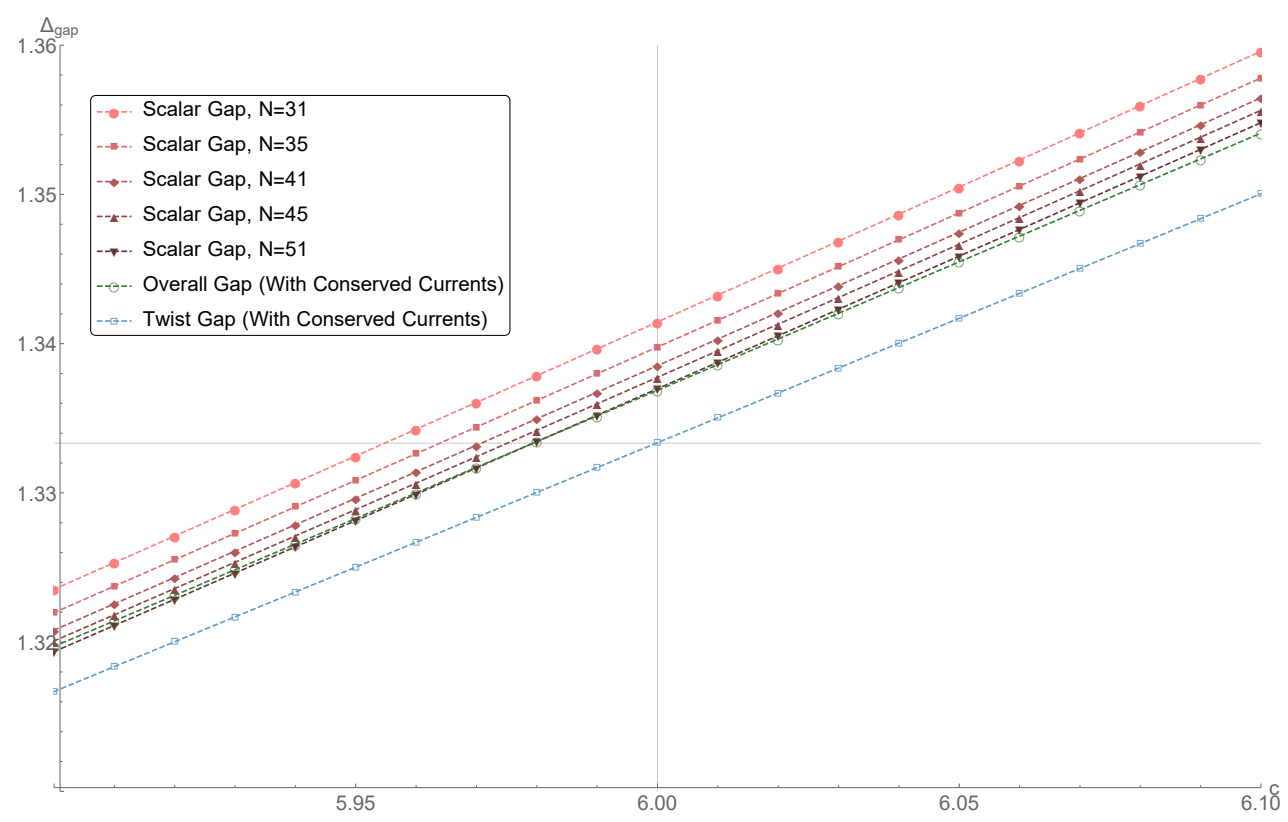

(a) $5.9 \leq c \leq 6.1$

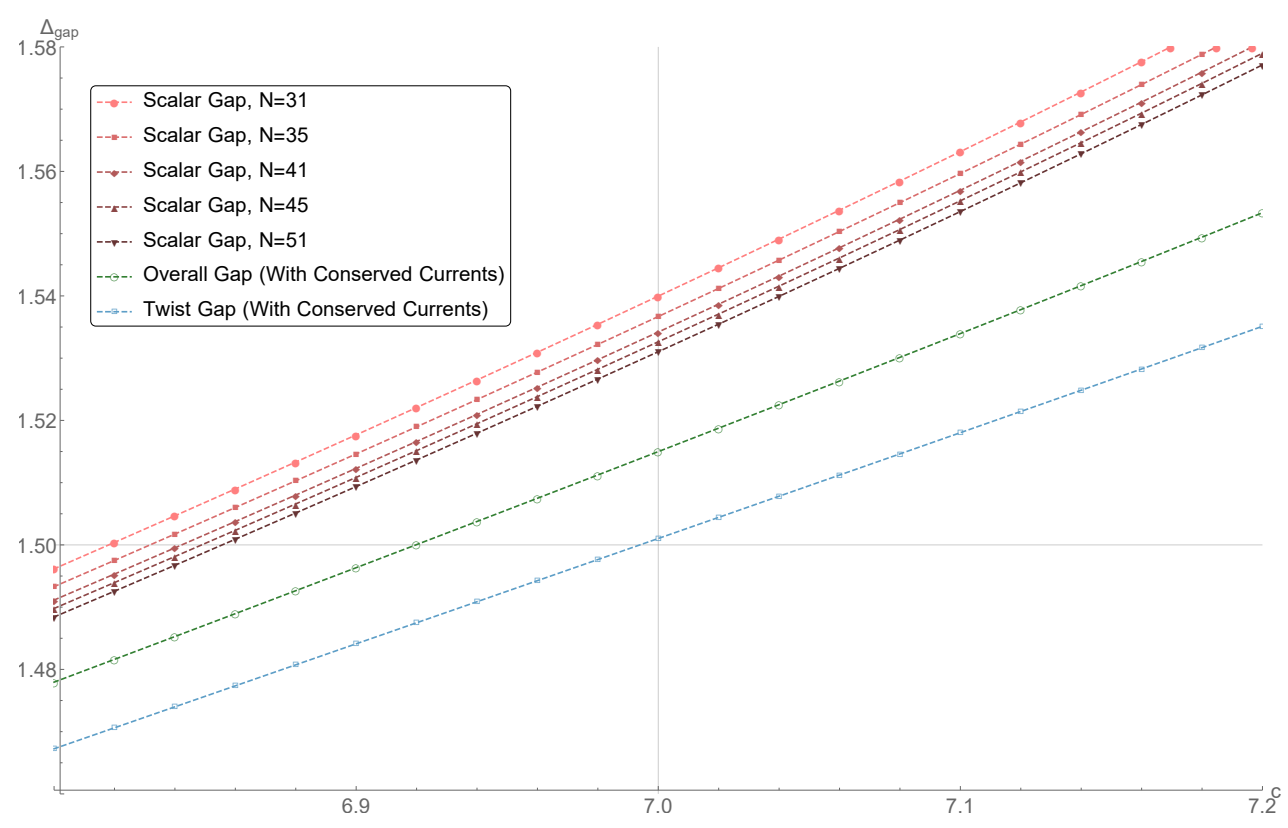

(b) $6.8 \leq c \leq 7.2$

Figure 5. Numerical upper bounds on scalar gap, overall gap and twist gap zoomed in around $c=6$ and 7 .

In figure 6 , we further explore the numerical upper bounds on the twist gap $\Delta_{t}$ for CFTs with conserved currents, by restricting the spins of the conserved currents. We consider the case without the spin- 1 current (only the spins $j \geq 2$ included), and also the case without $j=1,2$ current (only the spins $j \geq 3$ included).

We find 13 special points on the numerical upper bounds of $\Delta_{t}$ that may correspond to RCFTs, some of which are known and the others are conjectured, when $j \geq 1$ con- 


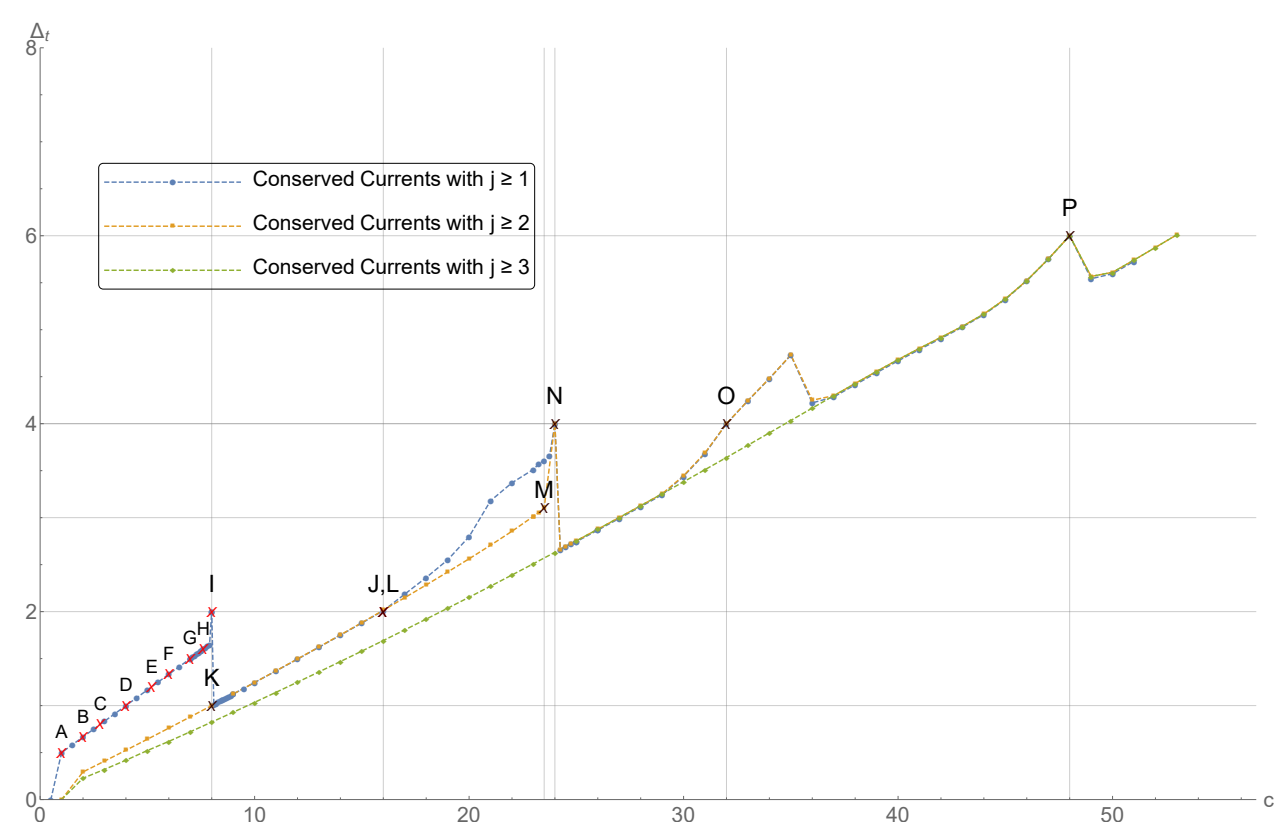

Figure 6. Numerical upper bounds on the twist gap in the range of $1 \leq c \leq 55$ with the conserved currents of $j \geq 1, j \geq 2$ and $j \geq 3$ included. Each $\mathbf{x}$-mark on the boundary refers to a certain RCFT in given in tables 3,4 .

served currents are included. Those RCFTs are summarized in table 3. We can further show that only these 13 RCFTs saturate the integer bounds on the degeneracies of the scalar primaries with dimension $\Delta=\Delta_{t}$. We will discuss this point in section 4 . If we exclude $j=1$ conserved current in the spectrum, the three additional RCFTs conjectured in [36], including the Baby Monster CFT, are realized at the numerical bounds. They are summarized in table 4.

\section{Spectroscopy}

\subsection{Spectrum analysis in modular bootstrap}

We discuss in this section how to constrain the degeneracy of primary operators above the vacuum in a hypothetical CFT when the numerical bound on the twist gap $\Delta_{t}$ is saturated [14]. Let us first start with upper bound on the degeneracy of the lowest scalar primaries of conformal dimension $\Delta=\Delta_{t}$. As long as $\Delta_{t}$ is below the numerical bound, there can exist certain linear functional $\beta$ such that

$$
\beta\left[\mathcal{Z}_{\frac{\Delta_{t}}{2}, \frac{\Delta_{t}}{2}}(\tau, \bar{\tau})\right]=1
$$

and

$$
\beta\left[\mathcal{Z}_{h, \bar{h}}(\tau, \bar{\tau})\right] \geq 0 \text { for }(h, \bar{h}) \neq\left(\frac{\Delta_{t}}{2}, \frac{\Delta_{t}}{2}\right), \quad \beta\left[\mathcal{Z}_{j}(\tau, \bar{\tau})\right] \geq 0
$$




\begin{tabular}{|c|c|c|c|c|}
\hline Label & $c$ & $\Delta_{t}$ & Maximal Degeneracy & Expected CFT \\
\hline A & 1 & $1 / 2$ & $\operatorname{deg}=4.000000$ & $\left(\widehat{A}_{1}\right)_{1}$ WZW model \\
\hline $\mathrm{B}$ & 2 & $2 / 3$ & $\operatorname{deg}=18.000000$ & $\left(\widehat{A}_{2}\right)_{1} \mathrm{WZW}$ model \\
\hline $\mathrm{C}$ & $14 / 5$ & $4 / 5$ & $\operatorname{deg}=49.000000$ & $\left(\widehat{G}_{2}\right)_{1}$ WZW model \\
\hline $\mathrm{D}$ & 4 & 1 & $\operatorname{deg}=192.0000000$ & $\left(\widehat{D}_{4}\right)_{1} \mathrm{WZW}$ model \\
\hline $\mathrm{E}$ & $26 / 5$ & $6 / 5$ & $\operatorname{deg}=676.000004$ & $\left(\widehat{F}_{4}\right)_{1} \mathrm{WZW}$ model \\
\hline $\mathrm{F}$ & 6 & $4 / 3$ & $\operatorname{deg}=1458.000091$ & $\left(\widehat{E}_{6}\right)_{1}$ WZW model \\
\hline G & 7 & $3 / 2$ & $\operatorname{deg}=3136.000011$ & $\left(\widehat{E}_{7}\right)_{1} \mathrm{WZW}$ model \\
\hline $\mathrm{H}$ & $38 / 5$ & $8 / 5$ & $\operatorname{deg}=3249.000405$ & $\left(\widehat{E}_{7 \frac{1}{2}}\right)_{1}$ WZW model \\
\hline I & 8 & 2 & $\operatorname{deg}=61504.00000$ & $\left(\widehat{E}_{8}\right)_{1}$ WZW model \\
\hline $\mathrm{J}$ & 16 & 2 & $\operatorname{deg}=246016.0000$ & $\left(\widehat{E}_{8} \times \widehat{E}_{8}\right)_{1}$ WZW model \\
\hline $\mathrm{N}$ & 24 & 4 & $\operatorname{deg}=38762915689.0000$ & Monster CFT \\
\hline $\mathrm{O}$ & 32 & 4 & $\operatorname{deg}=19461087009.0351$ & $" c=32$ ECFT" \\
\hline $\mathrm{P}$ & 48 & 6 & $\operatorname{deg}=1847926789775361.00$ & $c=48 \mathrm{ECFT}$ \\
\hline
\end{tabular}

Table 3. List of theories on the numerical boundary of $\Delta_{t}$. We include the conserved currents of $j \geq 1$ in spectrum.

\begin{tabular}{|c|c|c|c|c|}
\hline Label & $c$ & $\Delta_{t}$ & Maximal Degeneracy & Automorphism \\
\hline $\mathrm{K}$ & 8 & 1 & $\operatorname{deg}=496.000000$ & $O_{10}^{+}(2) .2$ \\
\hline $\mathrm{L}$ & 16 & 2 & $\operatorname{deg}=69255.00000$ & $O_{10}^{+}(2) .2^{16}$ \\
\hline $\mathrm{M}$ & $47 / 2$ & 3 & $\operatorname{deg}=19105641.071$ & Baby Monster \\
\hline
\end{tabular}

Table 4. List of theories on the numerical boundary of $\Delta_{t}$. We include the conserved currents of $j \geq 2$ in spectrum.

but acts negatively on $\mathcal{Z}_{\text {vac }}(\tau, \bar{\tau})$ to satisfy the modular constraint (3.5). Then, (4.1) and (4.2) imply

$$
\begin{aligned}
2 d_{\frac{\Delta_{t}}{2}, \frac{\Delta_{t}}{2}} & =-\beta\left[\mathcal{Z}_{\mathrm{vac}}\right]-\left\{\sum_{(h, \bar{h}) \neq\left(\frac{\Delta_{t}}{2}, \frac{\Delta_{t}}{2}\right)} d_{h, \bar{h}} \beta\left[\mathcal{Z}_{h, \bar{h}}+\overline{\mathcal{Z}}_{h, \bar{h}}\right]+\sum_{j} d_{h} \beta\left[\mathcal{Z}_{j}+\overline{\mathcal{Z}}_{j}\right]\right\} \\
& \leq-\beta\left[\mathcal{Z}_{\mathrm{vac}}(\tau, \bar{\tau})\right] .
\end{aligned}
$$

To obtain the upper bound on $d_{\frac{\Delta_{t}}{2}, \frac{\Delta_{t}}{2}}$, one thus need to solve an optimization problem of searching a linear functional $\beta$ that maximize

$$
\beta\left[\mathcal{Z}_{\mathrm{vac}}(\tau, \bar{\tau})\right]
$$


Let us denote such a linear functional by $\beta^{*}$. Then this leads to

$$
d_{\frac{\Delta_{t}}{2}, \frac{\Delta_{t}}{2}} \leq d_{\frac{\Delta_{t}}{2}, \frac{\Delta_{t}}{2}}^{*}=-\frac{1}{2} \beta^{*}\left[\mathcal{Z}_{\mathrm{vac}}(\tau, \bar{\tau})\right] .
$$

It will be shown later that the 16 RCFTs that lie on the numerical bounds on $\Delta_{t}$ saturate the degeneracy bound (4.5).

We can further determine the entire spectrum of a putative CFT, referred to as the extremal spectrum, when the degeneracy of the lowest primary scalar saturates the bound (4.5). This is because, when $d_{\frac{\Delta_{t}}{2}, \frac{\Delta_{t}}{2}}=d_{\frac{\Delta_{t}}{2}, \frac{\Delta_{t}}{2}}^{*}$, the first line of (4.3) becomes

$$
\begin{aligned}
0= & \sum_{(h, \bar{h}) \neq\left(\frac{\Delta_{t}}{2}, \frac{\Delta_{t}}{2}\right)} d_{h, \bar{h}}\left\{\beta^{*}\left[\mathcal{Z}_{h, \bar{h}}(\tau, \bar{\tau})\right]+\beta^{*}\left[\overline{\mathcal{Z}}_{h, \bar{h}}(\tau, \bar{\tau})\right]\right\} \\
& +\sum_{j} d_{j}\left\{\beta^{*}\left[\mathcal{Z}_{j}(\tau, \bar{\tau})\right]+\beta^{*}\left[\overline{\mathcal{Z}}_{j}(\tau, \bar{\tau})\right]\right\} .
\end{aligned}
$$

and thus

$$
\left.\beta^{*}\left[\mathcal{Z}_{h, \bar{h}}(\tau, \bar{\tau})\right]=0 \quad\left(\beta^{*}\left[\mathcal{Z}_{j, 0}(\tau, \bar{\tau})\right]\right]=0\right)
$$

for each primary in the extremal spectrum, i.e., $d_{h, \bar{h}} \neq 0\left(d_{j} \neq 0\right)$. Therefore, by examining the functional $\beta^{*}$ evaluated at the Virasoro weights $(h, \bar{h})$, one can determine which value of the weights are allowed. This analysis is initiated in [46], often called as the extremal functional method.

One can also find the upper bounds on the degeneracies $d_{h^{\prime}, \bar{h}^{\prime}}$ of the primaries in the extremal spectrum. This can be done by solving another optimization problem, that is to search for a linear functional $\beta_{\left(h^{\prime}, \bar{h}^{\prime}\right)}^{*}$ that

$$
\operatorname{maximize} \beta_{\left(h^{\prime}, \bar{h}^{\prime}\right)}^{*}\left[\mathcal{Z}_{\mathrm{vac}}(\tau, \bar{\tau})\right]
$$

such that

$$
\beta_{\left(h^{\prime}, \bar{h}^{\prime}\right)}^{*}\left[\mathcal{Z}_{h^{\prime}, \bar{h}^{\prime}}(\tau, \bar{\tau})\right]=1,
$$

and

$$
\beta_{\left(h^{\prime}, \bar{h}^{\prime}\right)}^{*}\left[\mathcal{Z}_{h, \bar{h}}(\tau, \bar{\tau})\right] \geq 0 \text { for }(h, \bar{h}) \neq\left(h^{\prime}, \bar{h}^{\prime}\right)
$$

Although it is not guaranteed that a CFT having the extremal spectrum always maximizes the degeneracies at all weights, it is still interesting to ask if there exist such CFTs. We will see soon in the next subsection that 16 RCFTs that are realized on the numerical bounds of $\Delta_{t}$ indeed saturate the upper bounds on the degeneracies of all the primaries in the extremal spectrum. 

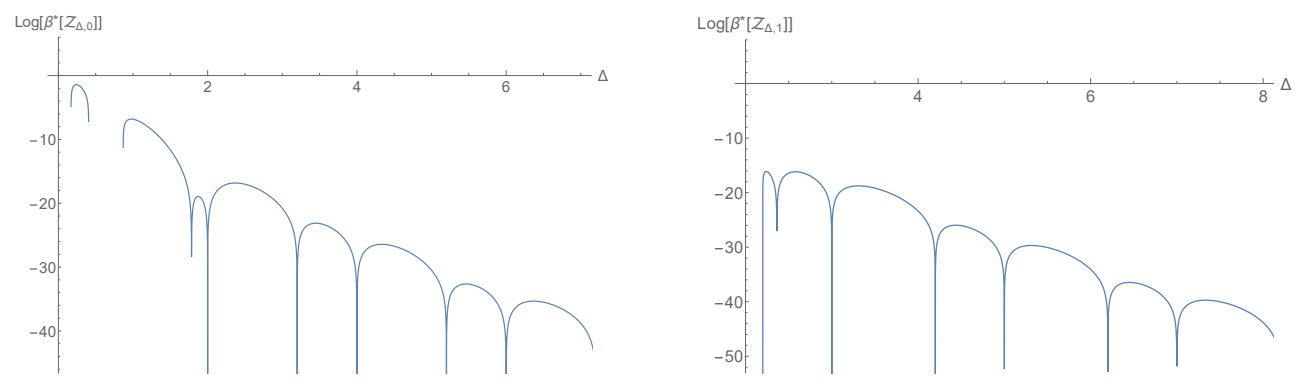

Figure 7. The extremal functional $\beta^{*}$ acting on spin-0 (left) and spin-1 (right) primaries at $c=\frac{26}{5}$ and $\Delta_{t}=\frac{6}{5}$.

\begin{tabular}{|c|c|c|c|c|c|}
\hline$(h, \bar{h})$ & Max. Deg & $(h, \bar{h})$ & Max. Deg & $(h, \bar{h})$ & Max. Deg \\
\hline$\left(\frac{3}{5}, \frac{3}{5}\right)$ & 676.0000 & $(1,1)$ & 2704.0000 & $(1,0)$ & 52.00028 \\
\hline$\left(\frac{3}{5}, \frac{8}{5}\right)$ & 7098.0001 & $(2,1)$ & 16848.001 & $(2,0)$ & 324.0007 \\
\hline$\left(\frac{3}{5}, \frac{13}{5}\right)$ & 35802.002 & $(3,1)$ & 80444.061 & $(3,0)$ & 1547.0091 \\
\hline$\left(\frac{8}{5}, \frac{8}{5}\right)$ & 74529.0001 & $(2,2)$ & 104976.005 & $(4,0)$ & 5499.0126 \\
\hline
\end{tabular}

Table 5. The maximum value of degeneracies for low-lying states in a putative CFT with $c=\frac{26}{5}$.

\subsection{WZW models with Deligne's exceptional series}

In this subsection, we utilize the extremal functional method (EFM) to investigate hypothetical CFTs on $\Delta_{t}=\Delta_{t}^{*}$ having the maximal degeneracies at all weights in the extremal spectrum. It turns out that the WZW models with level one for Deligne's exceptional series are the CFTs of such type. Among them, the spectrum of WZW models for $\mathfrak{g}=\widehat{A}_{1}, \widehat{A}_{2}, \widehat{G}_{2}, \widehat{D}_{4}$ and $\widehat{E}_{8}$ are also shown to agree with the extremal spectrum of CFTs on $\Delta_{s}=\Delta_{s}^{*}$ at $c=1,2, \frac{14}{5}, 4$ and 8 [14]. We present below our numerical spectral data at $c=\frac{26}{5}, 6,7, \frac{38}{5}$ from which we identify the CFTs of our interest with the WZW models for $\mathfrak{g}=\widehat{F}_{4}, \widehat{E}_{6}, \widehat{E}_{7}$ and also with the mysterious $\widehat{E}_{7 \frac{1}{2}}$.

- Spectrum analysis for the $\left(\widehat{F}_{4}\right)_{1}$ WZW model

We apply the EFM to a hypothetical CFT with $c=\frac{26}{5}$. The results illustrated in figure 7 suggest that the extremal spectrum of spin- 0 and spin- 1 have the conformal dimensions $\Delta_{j=0}=\left\{\frac{6}{5}+2 n, 2+2 n\right\}$ and $\Delta_{j=1}=\left\{\frac{11}{5}+2 n, 3+2 n\right\}$ for $n \in \mathbb{Z}_{\geq 0}$.

We utilize the linear functionals $\beta_{h, \bar{h}}^{*}$ in (4.8)-(4.10) to obtain the maximal degeneracies of low-lying primaries in the extremal spectrum, listed in table 5 . It implies that in terms of the Virasoro characters, the partition function of a putative CFT of 

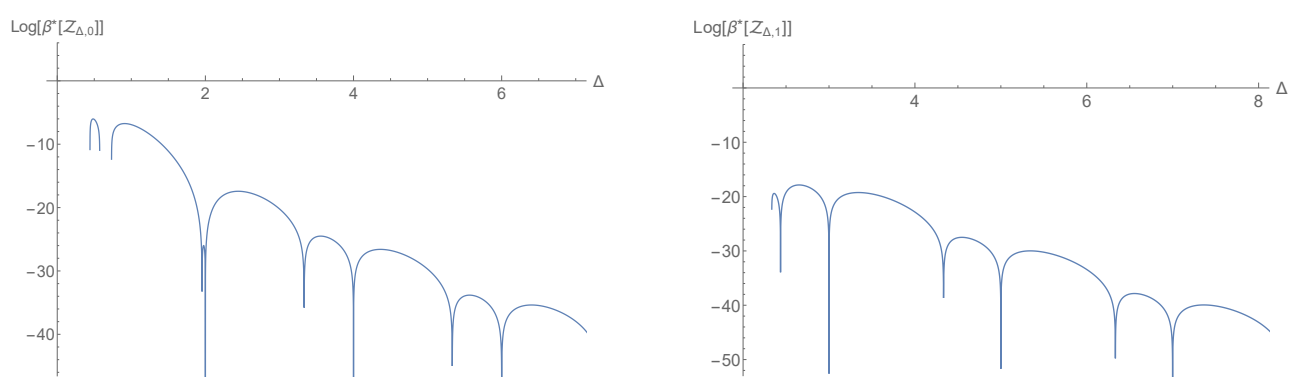

Figure 8. The extremal functional $\beta^{*}$ acting on spin-0 (left) and spin-1 (right) primaries at $c=6$ and $\Delta_{t}=\frac{4}{3}$.

our interest can be decomposed into the following form.

$$
\begin{aligned}
Z_{c=\frac{26}{5}}(\tau, \bar{\tau})= & \chi_{0}(\tau) \bar{\chi}_{0}(\bar{\tau})+676 \chi_{\frac{3}{5}}(\tau) \bar{\chi}_{\frac{3}{5}}(\bar{\tau})+7098\left(\chi_{\frac{3}{5}}(\tau) \bar{\chi}_{\frac{8}{5}}(\bar{\tau})+\text { c.c. }\right) \\
& +2704 \chi_{1}(\tau) \bar{\chi}_{1}(\bar{\tau})+16848\left(\chi_{2}(\tau) \bar{\chi}_{1}(\bar{\tau})+\text { c.c. }\right)+104976 \chi_{2}(\tau) \bar{\chi}_{2}(\bar{\tau}) \\
& +52\left(\chi_{1}(\tau) \bar{\chi}_{0}(\bar{\tau})+\text { c.c. }\right)+324\left(\chi_{2}(\tau) \bar{\chi}_{0}(\bar{\tau})+\text { c.c. }\right)+\cdots
\end{aligned}
$$

The affine character of $\left(\widehat{F}_{4}\right)_{1}$ is known to agree with the solution (2.13) to the second order modular differential equation (2.7) with $c=\frac{26}{5}$,

$$
\begin{aligned}
& f_{\text {vac }}^{c=\frac{26}{5}}(\tau) \equiv \chi_{[1 ; 0,0,0,0]}^{c=\frac{26}{5}}(\tau)=q^{-\frac{13}{60}}\left(1+52 q+377 q^{2}+1976 q^{3}+\mathcal{O}\left(q^{4}\right)\right), \\
& f_{\frac{3}{5}}^{c=\frac{26}{5}}(\tau) \equiv \chi_{[0 ; 0,0,0,1]}^{c=\frac{26}{5}}(\tau)=q^{\frac{3}{5}-\frac{13}{60}}\left(26+299 q+1702 q^{2}+7475 q^{3}+\mathcal{O}\left(q^{4}\right)\right),
\end{aligned}
$$

where the overall constant $a_{0}$ of (2.13) is now fixed by the dimension of fundamental representation of $F_{4}$.

Using this affine character, one can simplify the modular invariant partition function (4.11) as

$$
Z_{c=\frac{26}{5}}(\tau, \bar{\tau})=\left|f_{\text {vac }}^{F_{4}}(\tau)\right|^{2}+\left|f_{\frac{3}{5}}^{F_{4}}(\tau)\right|^{2},
$$

which perfectly agree with the modular invariant partition function of $\left(\widehat{F}_{4}\right)_{1}$ WZW model [34]. Therefore, we identify the putative CFT at $c=\frac{26}{5}$ with the $\left(\widehat{F}_{4}\right)_{1}$ WZW model.

- Spectrum analysis for the $\left(\widehat{E}_{6}\right)_{1}$ WZW model

Using the linear functional (4.7), we can learn that the extremal spectrum with $c=6$ and $\Delta_{t}=\frac{4}{3}$ contains the scalar primaries of $\Delta_{j=0}=\left\{\frac{4}{3}+2 n, 2+2 n\right\}$ and spin-one primaries of $\Delta_{j=1}=\left\{\frac{7}{3}+2 n, 3+2 n\right\}(n \geq 0)$, as depicted in figure 8. We also summarize the maximal degeneracies of various primaries in the extremal spectrum in table 6 .

We can express the partition function of a CFT that contains primaries in table 6 in terms of two solutions to (2.7) with $c=6$ as follows,

$$
Z_{c=6}(\tau, \bar{\tau})=f_{\text {vac }}^{c=6}(\tau) \bar{f}_{\text {vac }}^{c=6}(\bar{\tau})+2 f_{\frac{2}{3}}^{c=6}(\tau) \bar{f}_{\frac{2}{3}}^{c=6}(\bar{\tau}),
$$




\begin{tabular}{|c|c|c|c|c|c|}
\hline$(h, \bar{h})$ & Max. Deg & $(h, \bar{h})$ & Max. Deg & $(h, \bar{h})$ & Max. Deg \\
\hline$\left(\frac{2}{3}, \frac{2}{3}\right)$ & 1458.0001 & $(1,1)$ & 6084.0001 & $(1,0)$ & 78.00023 \\
\hline$\left(\frac{2}{3}, \frac{5}{3}\right)$ & 18954.003 & $(2,1)$ & 50700.004 & $(2,0)$ & 650.0012 \\
\hline$\left(\frac{2}{3}, \frac{8}{3}\right)$ & 112266.08 & $(3,1)$ & 278850.00 & $(3,0)$ & 3575.010 \\
\hline$\left(\frac{5}{3}, \frac{5}{3}\right)$ & 246402.001 & $(2,2)$ & 422500.05 & $(4,0)$ & 14806.03 \\
\hline
\end{tabular}

Table 6. The maximum value of degeneracies for low-lying states in a putative CFT with $c=6$.
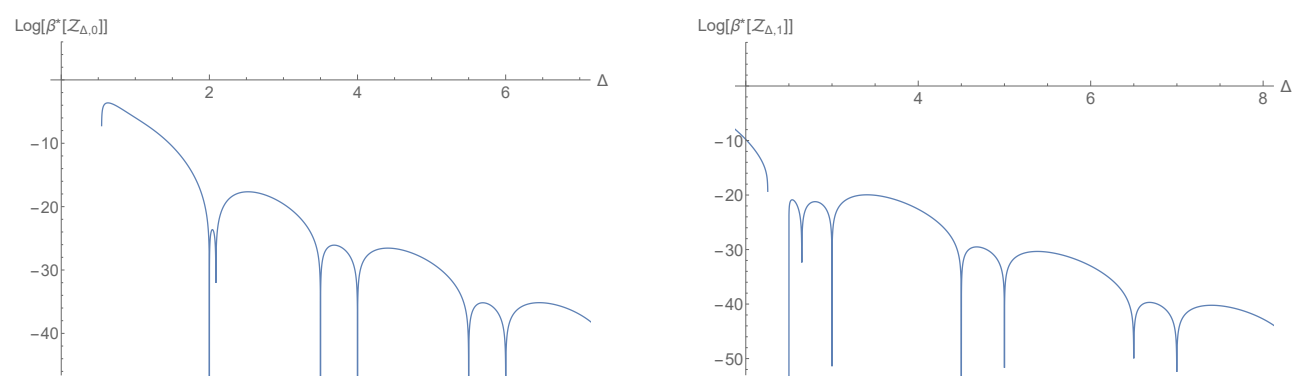

Figure 9. The extremal functional $\beta^{*}$ acting on spin-0 (left) and spin-1 (right) primaries at $c=7$ and $\Delta_{t}=\frac{3}{2}$.

where

$$
\begin{aligned}
& f_{\text {vac }}^{c=6}(\tau)=q^{-\frac{1}{4}}\left(1+78 q+729 q^{2}+4382 q^{3}+\mathcal{O}\left(q^{4}\right)\right) \\
& f_{\frac{2}{3}}^{c=6}(\tau)=q^{\frac{2}{3}-\frac{1}{4}}\left(27+378 q+2484 q^{2}+12312 q^{3}+\mathcal{O}\left(q^{4}\right)\right)
\end{aligned}
$$

Furthermore, two solutions $f_{\text {vac }}^{c=6}(\tau)$ and $f_{\frac{2}{3}}^{c=6}(\tau)$ can be identified as the affine characters of $\widehat{E}_{6}$

$$
\begin{aligned}
& f_{\mathrm{vac}}^{c=6}(\tau)=\chi_{[1 ; 0,0,0,0,0,0]}^{c=6}(\tau) \\
& f_{\frac{2}{3}}^{c=6}(\tau)=\chi_{[0 ; 1,0,0,0,0,0]}^{c=6}(\tau)=\chi_{[0 ; 0,0,0,0,1,0]}^{c=6}(\tau)
\end{aligned}
$$

Here, two representations $[0 ; 1,0,0,0,0,0]$ and $[0 ; 0,0,0,0,1,0]$ are complex conjugate to each other, and their characters are indistinguishable unless we turn on additional chemical potentials for the Cartan parts of the current algebra.

The partition function (4.14) then becomes the partition function of $\left(\widehat{E}_{6}\right)_{1}$ WZW model (2.17).

- Spectrum analysis for the $\left(\widehat{E}_{7}\right)_{1}$ WZW model

As depicted in figure 9, the extremal spectrum with $c=7$ and $\Delta_{t}=\frac{3}{2}$ contains spin-0 primaries of $\Delta_{j=0}=\left\{\frac{3}{2}+2 n, 2+2 n\right\}$ and spin-1 primaries of $\Delta_{j=1}=\left\{\frac{5}{2}+2 n, 3+2 n\right\}$ $\left(n \in \mathbb{Z}_{\geq 0}\right)$. The maximal degeneracies at various weights in the extremal spectrum are listed in table 7 . 


\begin{tabular}{|c|c|c|c|c|c|}
\hline$(h, \bar{h})$ & Max. Deg & $(h, \bar{h})$ & Max. Deg & $(h, \bar{h})$ & Max. Deg \\
\hline$\left(\frac{3}{4}, \frac{3}{4}\right)$ & 3136.0000 & $(1,1)$ & 17689.000 & $(1,0)$ & 133.00116 \\
\hline$\left(\frac{3}{4}, \frac{7}{4}\right)$ & 51072.000 & $(2,1)$ & 204687.00 & $(2,0)$ & 1539.0104 \\
\hline$\left(\frac{3}{4}, \frac{11}{4}\right)$ & 362880.00 & $(3,1)$ & 1344364.01 & $(3,0)$ & 10108.085 \\
\hline$\left(\frac{7}{4}, \frac{7}{4}\right)$ & 831744.01 & $(2,2)$ & 2368521.01 & $(4,0)$ & 49665.351 \\
\hline
\end{tabular}

Table 7. The maximum value of degeneracies for low-lying states in a putative CFT with $c=7$.

The solutions to (2.7) with $c=7$ are known to agree with the $\widehat{E}_{7}$ affine character with $a_{0}=56$

$$
\begin{aligned}
& f_{\mathrm{vac}}^{c=7}(\tau)=\chi_{[1 ; 0,0,0,0,0,0,0]}^{c=7}(\tau)=q^{-\frac{7}{24}}\left(1+133 q+1673 q^{2}+11914 q^{3}+\ldots\right), \\
& f_{\frac{3}{4}}^{c=7}(\tau)=\chi_{[0 ; 0,0,0,0,0,1,0]}^{c=7}(\tau)=q^{\frac{3}{4}-\frac{7}{24}}\left(56+968 q+7504 q^{2}+42616 q^{3}+\ldots\right) .
\end{aligned}
$$

It is straightforward to see that the partition function of $\left(\widehat{E}_{7}\right)_{1}$ WZW model $(2.17)$,

$$
Z_{c=7}(\tau, \bar{\tau})=\left|f_{\mathrm{vac}}^{c=7}(\tau)\right|^{2}+\left|f_{\frac{3}{4}}^{c=7}(\tau)\right|^{2}
$$

is consistent to the maximal degeneracies in table 7 .

- Spectrum analysis for the $\left(\widehat{E}_{7 \frac{1}{2}}\right)_{1}$ WZW model

It is shown in [28] that there is one more value of central charge $c=\frac{38}{5}$ where the all the coefficients of the two solutions to (2.7) become positive integers,

$$
\begin{aligned}
& f_{\mathrm{vac}}^{c=\frac{38}{5}}(\tau)=q^{-\frac{19}{60}}\left(1+190 q+2831 q^{2}+22306 q^{3}+129276 q^{4}+\mathcal{O}\left(q^{5}\right)\right), \\
& f_{\frac{4}{5}}^{c=\frac{38}{5}}(\tau)=q^{\frac{4}{5}-\frac{19}{60}}\left(57+1102 q+9367 q^{2}+57362 q^{3}+280459 q^{4}+\mathcal{O}\left(q^{5}\right)\right) .
\end{aligned}
$$

The non-vacuum character $f_{\text {vac }}^{c=\frac{38}{5}}(\tau)$ in (4.19) can arise from an affine Lie algebra if there is one with dimension 190. Interestingly, mathematicians have discovered that there is indeed such a Lie algebra called $E_{7 \frac{1}{2}}$, in an attempt to fill in a certain gap in the Deligne's exceptional series [47].

As illustrated in figure 10, the extremal spectrum contains scalar primaries of conformal dimension $\Delta_{j=0}=\left\{\frac{8}{5}+2 n, 2+2 n\right\}$ and spin-one primaries of conformal dimension $\Delta_{j=1}=\left\{\frac{13}{5}+2 n, 3+2 n\right\}\left(n \in \mathbb{Z}_{\geq 0}\right)$. As summarized in table 8 , they have positive integer maximal degeneracies.

It turns out that the modular invariant partition function of the a CFT that contains primaries in table 8 can be simply expressed as,

$$
Z_{c=\frac{38}{5}}(\tau, \bar{\tau})=f_{\mathrm{vac}}^{c=\frac{38}{5}}(\tau) \bar{f}_{\mathrm{vac}}^{c=\frac{38}{5}}(\bar{\tau})+f_{\frac{4}{5}}^{c=\frac{38}{5}}(\tau) \bar{f}_{\frac{4}{5}}^{c=\frac{38}{5}}(\bar{\tau}) .
$$



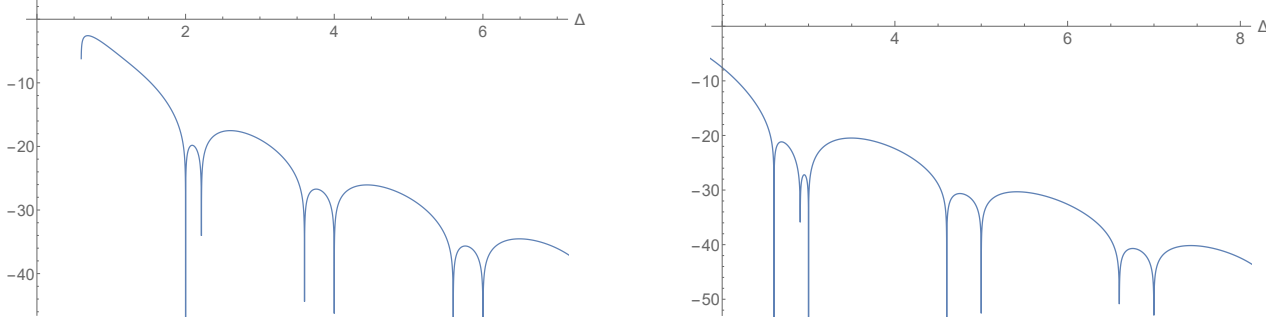

Figure 10. The extremal functional $\beta^{*}$ acting on spin-0 (left) and spin-1 (right) primaries at $c=\frac{38}{5}$ and $\Delta_{t}=\frac{8}{5}$.

\begin{tabular}{|c|c|c|c|c|c|}
\hline$(h, \bar{h})$ & Max. Deg & $(h, \bar{h})$ & Max. Deg & $(h, \bar{h})$ & Max. Deg \\
\hline$\left(\frac{4}{5}, \frac{4}{5}\right)$ & 3249.0004 & $(1,1)$ & 36100.000 & $(1,0)$ & 190.00412 \\
\hline$\left(\frac{4}{5}, \frac{9}{5}\right)$ & 59565.012 & $(2,1)$ & 501600.00 & $(2,0)$ & 2640.0481 \\
\hline$\left(\frac{9}{5}, \frac{9}{5}\right)$ & 1092025.06 & $(2,2)$ & 6969600.01 & $(3,0)$ & 19285.021 \\
\hline
\end{tabular}

Table 8. The maximum value of degeneracies for low-lying states in a putative CFT at $c=\frac{38}{5}$. For these results, the maximum number of derivative is set to $N_{\max }=55$, while spin is truncated at $j_{\max }=40$.
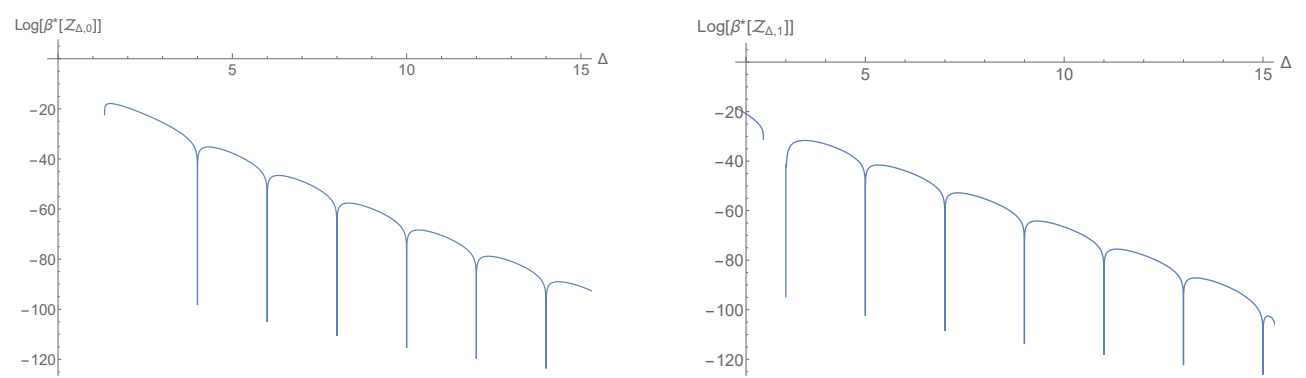

Figure 11. The extremal functional $\beta^{*}$ acting on spin-0 (left) and spin-1 (right) primaries at $c=16$ and $\Delta_{t}=2$.

Based on this observation, we suspect that there may exist a RCFT at $c=\frac{38}{5}$ where $f_{\text {vac }}^{c=\frac{38}{5}}(\tau)$ and $f_{\frac{4}{5}}^{c=\frac{38}{5}}(\tau)$ in $(4.19)$ can be understood as the characters of $\left(\widehat{E}_{7 \frac{1}{2}}\right)_{1} .^{5}$

\section{- Spectrum analysis for $\left(\widehat{E}_{8} \times \widehat{E}_{8}\right)_{1}$ WZW model}

The extremal spectrum of a putative CFT with $\left(c=16, \Delta_{t}=2\right)$ and their maximal degeneracies are presented in figure 11 and table 9, respectively. The partition

\footnotetext{
${ }^{5}$ It was shown in [33] that some of its fusion rules becomes negative. One can circumvent the puzzle by interchanging the two characters in (4.19). The new theory then becomes a non-unitary CFT with $c=-58 / 5$ which has 57 -fold vacua and primaries of negative dimension. Thus, the $c=-58 / 5$ theory cannot be understood as a standard CFT.
} 


\begin{tabular}{|c|c|c|c|}
\hline$(h, \bar{h})$ & Max. Deg & $(h, \bar{h})$ & Max. Deg \\
\hline$(1,1)$ & 246016.0000000000 & $(1,0)$ & 496.00000000000 \\
\hline$(2,1)$ & 34350480.00000000 & $(2,0)$ & 69255.0000000000 \\
\hline$(3,1)$ & 1014200960.000000 & $(3,0)$ & 2044760.00000000 \\
\hline$(2,2)$ & 4796255025.000000 & $(4,0)$ & 32485860.0000000 \\
\hline$(3,2)$ & 283219707600.0000 & $(5,0)$ & 357674373.000000 \\
\hline
\end{tabular}

Table 9. The maximum value of degeneracies for low-lying states in a putative CFT with $c=16$. Here the maximum number of derivative is set to $N_{\max }=55$, while the spin was truncated at $j_{\max }=40$.
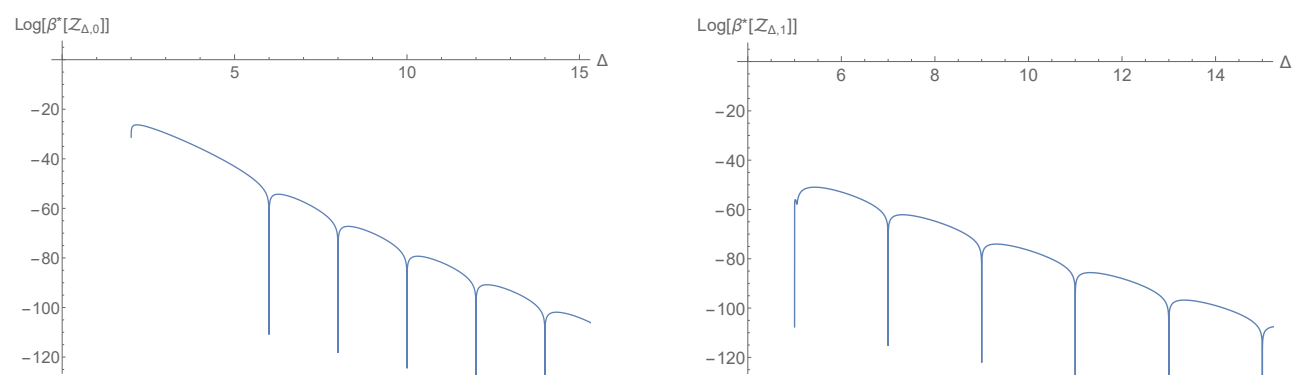

Figure 12. The extremal functional $\beta^{*}$ acting on spin-0 (left) and spin-1 (right) primaries at $c=24$ and $\Delta_{t}=4$.

function read off from table 9 . It can be written in a simple form

$$
Z_{c=16}(\tau, \bar{\tau})=j(\tau)^{\frac{2}{3}} \bar{j}(\bar{\tau})^{\frac{2}{3}},
$$

where $j(\tau)$ denotes the modular invariant $j$-function (2.24). The result (4.21) perfectly agrees with the partition function of the $\left(\widehat{E}_{8} \times \widehat{E}_{8}\right)_{1}$ WZW model.

\section{3 $c \geq 8$ RCFTs without Kac-Moody symmetry}

In the previous subsection, we uncovered that the WZW models with level one for Deligne's exceptional series maximize the degeneracies of the extremal spectrum at ten among sixteen special points on the numerical bound of $\Delta_{t}$, as depicted in figure 6 . It is known that the characters of such WZW models are the solutions to (2.7). In this subsection, we will show that the degeneracies of every extremal spectrum of certain RCFTs saturate their upper bound at the remaining $3+3$ points. It turns out that such RCFTs have characters that agree with the solutions to (2.8), and have no Kac-Moody symmetry but finite group symmetry of very large order. Such finite groups include the Monster and the Baby Monster groups.

- $c=24$ Monster CFT

Let us search for a hypothetical CFT with $c=24$ and $\Delta_{t}=4$ that contains the extremal spectrum, illustrated in figure 12, with the maximal degeneracies. From 


\begin{tabular}{|c|c|}
\hline$(h, \bar{h})$ & Max. Deg \\
\hline$(2,2)$ & 38762915689.000000 \\
\hline$(2,3)$ & 4192992837508.0000 \\
\hline$(2,4)$ & 165895451930858.000 \\
\hline$(3,3)$ & 453556927359376.000 \\
\hline$(3,4)$ & 17944946332265576.00 \\
\hline$(4,4)$ & 709990476262174276.00 \\
\hline
\end{tabular}

Table 10. The maximum value of degeneracies for low-lying states in a putative CFT with $c=24$. The maximum number of derivatives is set to $N_{\max }=55$, while the spin was truncated at $j_{\max }=40$.
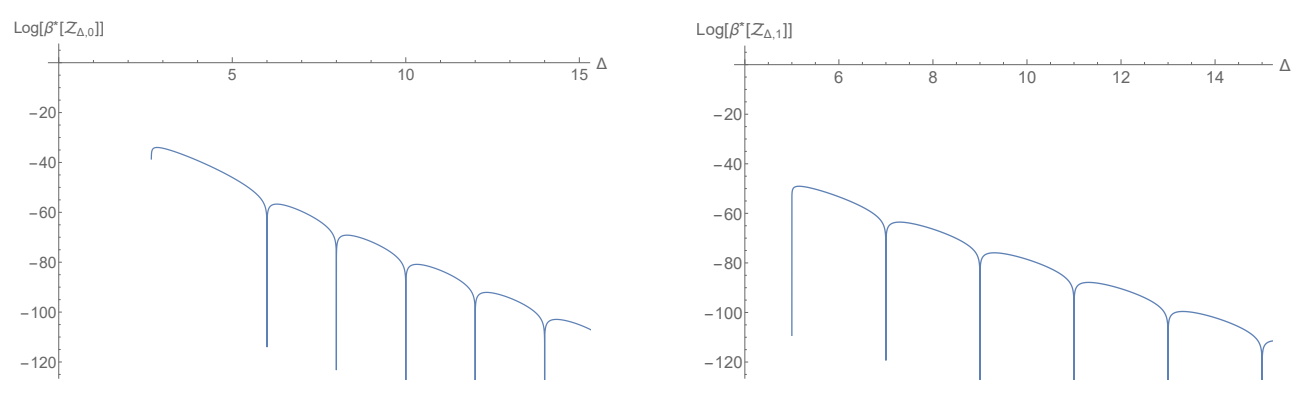

Figure 13. The extremal functional $\beta^{*}$ acting on spin-0 (left) and spin-1 (right) primaries at $c=32$ and $\Delta_{t}=4$.

table 10, we find its partition function can be written as

$$
\begin{aligned}
Z_{c=24}(\tau, \bar{\tau})= & \chi_{0}(\tau) \bar{\chi}_{0}(\bar{\tau})+\left(4192992837508 \chi_{2}(\tau) \bar{\chi}_{3}(\bar{\tau})+\text { c.c. }\right) \\
& +38762915689 \chi_{2}(\tau) \bar{\chi}_{2}(\bar{\tau})+453556927359376 \chi_{3}(\tau) \bar{\chi}_{3}(\bar{\tau}) \\
& +\left(165895451930858 \chi_{2}(\tau) \bar{\chi}_{4}(\bar{\tau})+\text { c.c. }\right)+\cdots \\
& +\left(196883 \chi_{2}(\tau) \bar{\chi}_{0}(\bar{\tau})+\text { c.c. }\right)+\cdots \\
= & (j(\tau)-744)(\bar{j}(\bar{\tau})-744) .
\end{aligned}
$$

Therefore we identify the putative CFT of our interest as the Monster CFT of [30,37].

- "c $=32$ Extremal CFT"

The extremal spectrum with $c=32$ and $\Delta_{t}=4$ have scalar primaries of $\Delta_{j=0}=\{4+2 n\}$ and spin-one primaries of $\Delta_{j=1}=\{5+2 n\}$ for $n \in \mathbb{Z}_{\geq 0}$, as depicted in figure 13. The upper bounds on degeneracies of the extremal spectrum are summarized in table 11. One can easily see that the partition function of the $c=32$ extremal CFT given below,

$$
Z_{c=32}(\tau, \bar{\tau})=\left(j(\tau)^{\frac{4}{3}}-992 j(\tau)^{\frac{1}{3}}\right)\left(\bar{j}(\bar{\tau})^{\frac{4}{3}}-992 \bar{j}(\bar{\tau})^{\frac{1}{3}}\right),
$$

is consistent with the maximal degeneracies as in table 11 . 


\begin{tabular}{|c|c|}
\hline$(h, \bar{h})$ & Max. Deg \\
\hline$(2,2)$ & 19461087009.000000000 \\
\hline$(2,3)$ & 9652699156464.0000004 \\
\hline$(2,4)$ & 966591762372600.00002 \\
\hline$(3,3)$ & 4787738781606144.0000 \\
\hline$(3,4)$ & 479429514136809600.01 \\
\hline$(4,4)$ & 48008604794505640003.18 \\
\hline
\end{tabular}

Table 11. The maximum value of degeneracies for low-lying states in putative CFT with $c=32$. The maximum number of derivatives is set to $N_{\max }=55$, while the spin is truncated at $j_{\max }=40$.

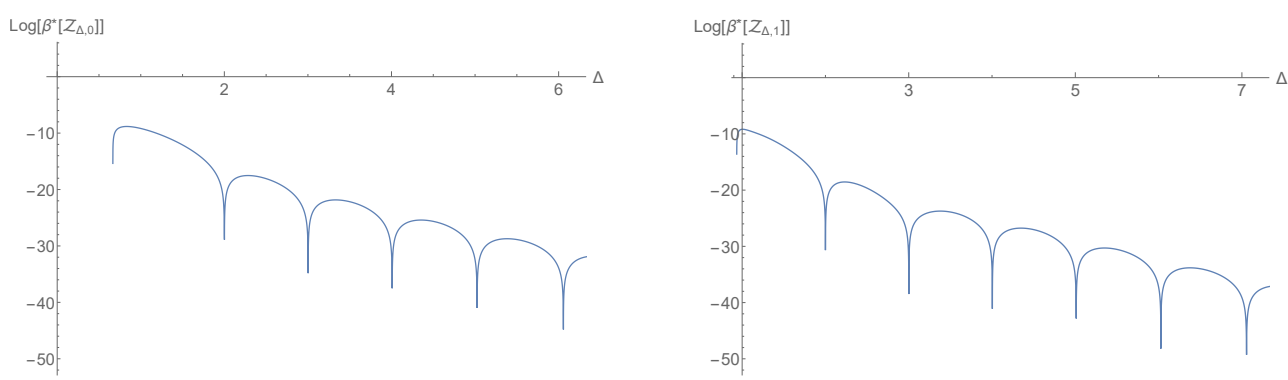

Figure 14. The extremal functional $\beta^{*}$ acting on spin-0 (left) and spin-1 (right) primaries at $c=8$ and $\Delta_{t}=1$, without the spin- 1 conserved current.

As discussed in [35], the vertex operator algebra giving the above partition function can be constructed as a $\mathbf{Z}_{2}$ orbifold of free bosons on an extremal self-dual lattice of rank 32. However, the classification and the automorphism group of these lattices have been poorly understood.

- $c=8$ RCFT without Kac-Moody symmetry

Note first that the CFT of our interest does not contain spin-one currents at all. Applying the EFM, we investigate the spin-0 and spin-1 extremal spectrum of a CFT with $\left(c=8, \Delta_{t}=1\right)$. Their conformal dimensions can be read from figure 14, $\Delta_{j=0}=\{1+n\}$ and $\Delta_{j=1}=\{2+n\}$ for $n \in \mathbb{Z}_{\geq 0}$. We also analyze the maximal degeneracies of the extremal spectrum, summarized in table 12. These results suggest that the partition function of a putative CFT with $\left(c=8, \Delta_{t}=1\right)$ but no Kac-Moody symmetry admits the character decomposition as

$$
\begin{aligned}
Z_{c=8}(\tau, \bar{\tau})= & \chi_{0}(\tau) \bar{\chi}_{0}(\bar{\tau})+496 \chi_{\frac{1}{2}}(\tau) \bar{\chi}_{\frac{1}{2}}(\bar{\tau})+17360\left(\chi_{\frac{1}{2}}(\tau) \bar{\chi}_{\frac{3}{2}}(\bar{\tau})+\text { c.c. }\right) \\
& +33728 \chi_{1}(\tau) \bar{\chi}_{1}(\bar{\tau})+505920\left(\chi_{2}(\tau) \bar{\chi}_{1}(\bar{\tau})+\text { c.c. }\right)+7612825 \chi_{2}(\tau) \bar{\chi}_{2}(\bar{\tau}) \\
& +155\left(\chi_{2}(\tau) \bar{\chi}_{0}(\bar{\tau})+\text { c.c. }\right)+868\left(\chi_{3}(\tau) \bar{\chi}_{0}(\bar{\tau})+\text { c.c. }\right)+\cdots
\end{aligned}
$$




\begin{tabular}{|c|c|c|c|c|c|}
\hline$(h, \bar{h})$ & Max. Deg & $(h, \bar{h})$ & Max. Deg & $(h, \bar{h})$ & Max. Deg \\
\hline$\left(\frac{1}{2}, \frac{1}{2}\right)$ & 496.0000000 & $(1,1)$ & 33728.00000 & $(2,0)$ & 155.000000 \\
\hline$\left(\frac{1}{2}, \frac{3}{2}\right)$ & 17360.00000 & $(2,1)$ & 505920.0000 & $(3,0)$ & 868.000000 \\
\hline$\left(\frac{3}{2}, \frac{3}{2}\right)$ & 607600.0009 & $(2,2)$ & 7612825.000 & $(4,0)$ & 5610.00000 \\
\hline
\end{tabular}

Table 12. The maximum value of degeneracies for low-lying states in putative CFT with $c=8$, without the spin-1 conserved current. The maximum number of derivatives is set to $N_{\max }=55$, while the spin is truncated at $j_{\max }=40$.

Using the solutions to (2.8) with $c=8$,

$$
\begin{aligned}
& f_{\text {vac }}^{c=8}(\tau)=q^{-\frac{1}{3}}\left(1+156 q^{2}+1024 q^{3}+6780 q^{4}+\mathcal{O}\left(q^{5}\right)\right), \\
& f_{\frac{1}{2}}^{c=8}(\tau)=q^{\frac{1}{2}-\frac{8}{24}}\left(1+36 q+394 q^{2}+2776 q^{3}+15155 q^{4}+\mathcal{O}\left(q^{5}\right)\right), \\
& f_{1}^{c=8}(\tau)=q^{1-\frac{8}{24}}\left(1+16 q+136 q^{2}+832 q^{3}+4132 q^{4}+\mathcal{O}\left(q^{5}\right)\right)
\end{aligned}
$$

it is straightforward to check that (4.24) can be recast into the following form,

$$
Z_{c=8}(\tau, \bar{\tau})=f_{\text {vac }}^{c=8}(\tau) f_{\text {vac }}^{c=8}(\bar{\tau})+496 f_{\frac{1}{2}}^{c=8}(\tau) \bar{f}_{\frac{1}{2}}^{c=8}+33728 f_{1}^{c=8}(\tau) \bar{f}_{1}^{c=8}(\bar{\tau}) .
$$

It is discussed in [35] that the automorphism group of the chiral CFT (more precisely, vertex operator algebra) having the vacuum character $f_{\text {vac }}^{c=8}(\tau)$ of $(4.25)$ is the finite group of Lie type, $O_{10}^{+}(2) .2$. We use the GAP package [48] to obtain the dimensions of the irreducible representations of $O_{10}^{+}(2)$, some of which are $155,340,868,2108$, $7905,14756,31620,55335,73780,505920,1048576,1422900$. Indeed, one can see that various coefficients in the character decomposition (4.24) can be expressed as sums of those dimensions

$$
\begin{aligned}
& 155, \quad 496=1+155+340, \quad 868, \\
& 17360=1+155+340+2108+14756, \\
& 33728=2108+31620, \quad 505920
\end{aligned}
$$

\section{- $c=16$ RCFT without Kac-Moody symmetry}

Let us search for a hypothetical CFT with $\left(c=16, \Delta_{t}=2\right)$ that does not have the Kac-Moody symmetry. The extremal spectrum of such a CFT and their upper bound of degeneracies are illustrated in figure 15 and table 13. These results imply that the partition function of the CFT of our interest can be expanded as

$$
\begin{aligned}
Z_{c=16}(q, \bar{q})= & \chi_{0}(\tau) \bar{\chi}_{0}(\bar{\tau})+32505856 \chi_{\frac{3}{2}}(\tau) \bar{\chi}_{\frac{3}{2}}(\bar{\tau})+1657798656\left(\chi_{\frac{3}{2}}(\tau) \bar{\chi}_{\frac{5}{2}}(\bar{\tau})+\text { c.c. }\right) \\
& +134912 \chi_{1}(\tau) \bar{\chi}_{1}(\bar{\tau})+18213120\left(\chi_{2}(\tau) \bar{\chi}_{1}(\bar{\tau})+\text { c.c. }\right) \\
& +2295\left(\chi_{2}(\tau) \bar{\chi}_{0}(\bar{\tau})+\text { c.c. }\right)+63240\left(\chi_{3}(\tau) \bar{\chi}_{0}(\bar{\tau})+\text { c.c. }\right)+\cdots, \\
= & f_{\operatorname{vac}}^{c=16}(\tau) \bar{f}_{\operatorname{vac}}^{c=16}(\bar{\tau})+134912 f_{1}^{c=16}(\tau) \bar{f}_{1}^{c=16}(\bar{\tau})+32505856 f_{\frac{3}{2}}^{c=16}(\tau) \bar{f}_{\frac{3}{2}}^{c=16}(\bar{\tau}),
\end{aligned}
$$




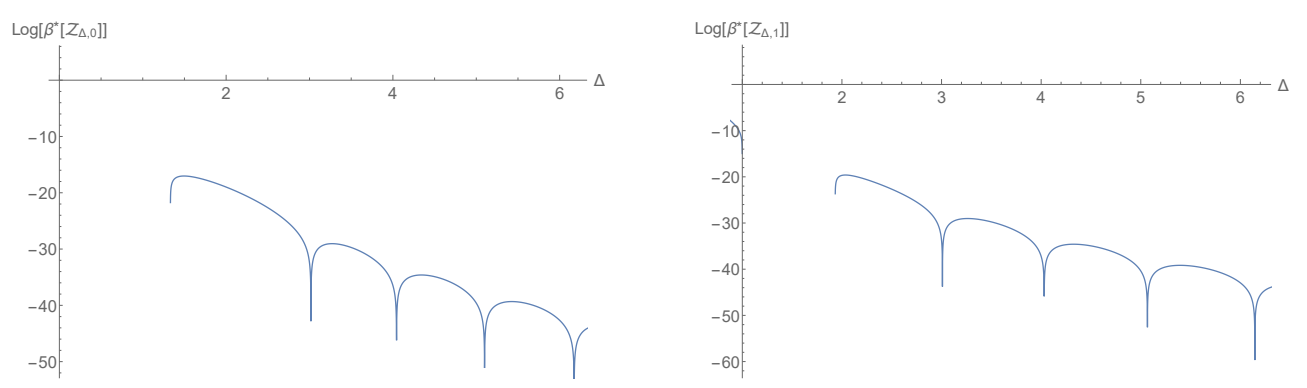

Figure 15. The extremal functional $\beta^{*}$ acting on spin-0(left) and spin-1(right) primaries at $c=16$ and $\Delta_{t}=2$, without the spin- 1 conserved current.

\begin{tabular}{|c|c|c|c|c|c|}
\hline$(h, \bar{h})$ & Max. Deg & $(h, \bar{h})$ & Max. Deg & $(h, \bar{h})$ & Max. Deg \\
\hline$\left(\frac{3}{2}, \frac{3}{2}\right)$ & 32505856.0032 & $(1,1)$ & 134912.0000 & $(2,0)$ & 2295.00000 \\
\hline$\left(\frac{3}{2}, \frac{5}{2}\right)$ & 1657798656.0001 & $(2,1)$ & 18213120.00 & $(3,0)$ & 63240.0000 \\
\hline$\left(\frac{3}{2}, \frac{7}{2}\right)$ & 34228666368.005 & $(2,2)$ & 2464038225.003 & $(4,0)$ & 1017636.00 \\
\hline
\end{tabular}

Table 13. This table summarizes the maximal degeneracy of first few states in gapped $c=16$ CFT. The total number of derivatives is set to $N_{\max }=55$, while the spin is truncated at $j_{\max }=40$.

where $f_{\text {vac }}^{c=16}(\tau), f_{1}^{c=16}(\tau)$ and $f_{\frac{3}{2}}^{c=16}(\tau)$ are solutions to $(2.8)$ with $c=16$. Note that various coefficients in the above decomposition of (4.28) can be written as sum of the dimensions of irreducible representations of $O_{10}^{+}(2)$,

$$
\begin{aligned}
2295 & =1+186+2108, \quad 63240=55335+7905, \\
134912 & =186+340+868+22848+110670, \\
18213120 & =12 \times 1422900+1048576+73780+14756+868+340 .
\end{aligned}
$$

This observation suggests that the above putative CFT with $\left(c=16, \Delta_{t}=2\right)$ but no Kac-Moody symmetry may have the $O_{10}^{+}(2)$ symmetry.

- Baby Monster CFT with $c=\frac{47}{2}$

In [36], the three solutions to (2.8) with $c=\frac{47}{2}$ are shown to be same as the characters of the Baby Monster vertex operator algebra [31],

$$
\begin{aligned}
& f_{\mathrm{vac}}^{c=\frac{47}{2}}(\tau)=\chi_{\mathrm{VB}_{(0)}^{\natural}}=q^{-\frac{47}{48}}\left(1+96256 q^{2}+9646891 q^{3}+366845011 q^{4}+\cdots\right) \\
& f_{\frac{3}{2}}^{c=\frac{47}{2}}(\tau)=\chi_{\mathrm{VB}_{(1)}^{\natural}}=q^{\frac{3}{2}-\frac{47}{48}}\left(4371+1143745 q+64680601 q^{2}+\cdots\right) \\
& f_{\frac{31}{16}}^{c=\frac{47}{2}}(\tau)=\chi_{\mathrm{VB}_{(2)}^{\natural}}=q^{\frac{31}{16}-\frac{47}{48}}\left(96256+10602496 q+420831232 q^{2}+\cdots\right) .
\end{aligned}
$$

We observe in figure 6 that the numerical bound at $c=\frac{47}{2}$ is given by $\Delta_{t}^{*}=3,{ }^{6}$ which is consistent with the character $f_{3 / 2}^{c=47 / 2}(\tau)$ of $(4.30)$.

\footnotetext{
${ }^{6}$ As shown in table 14, the upper bound on $\Delta_{t}$ at $c=\frac{47}{2}$ is approaching to 3 as we increase the total number of derivatives $N_{\max }$.
} 


\begin{tabular}{|c|c|c|}
\hline$N_{\max }$ & $\Delta_{t}$ & Max. degeneracy of $(h, \bar{h})=\left(\frac{3}{2}, \frac{3}{2}\right)$ \\
\hline 41 & 3.102 & 20633319.646029717379 \\
\hline 51 & 3.058 & 20060048.798539029429 \\
\hline 61 & 3.034 & 19728535.695677188476 \\
\hline 71 & 3.023 & 19597158.910830818660 \\
\hline 81 & 3.016 & 19499859.838240040877 \\
\hline
\end{tabular}

Table 14. The degeneracy upper bound of weight $(h, \bar{h})=\left(\frac{3}{2}, \frac{3}{2}\right)$ primary, for various $N_{\max }$.

Therefore, it is natural to expect that the corresponding CFT with $c=\frac{47}{2}$ and $\Delta_{t}=3$ has the Baby Monster symmetry. To support this hypothesis further, we analyze the maximal degeneracies of the scalar primary of $\Delta=3$, summarized in table 14. It appears that the upper bound is indeed converging to $4371^{2}=19105641$ as is predicted from the character. However, the convergence of bound is not fast enough as the order of derivative $N_{\max }$ is increased.

To circumvent the above numerical difficulty, we employ an alternative strategy of adding low-lying discrete spectrum in the semi-definite programming (4.8)-(4.10) by hand. More precisely, let us assume that the partition function of the Baby Monster $\mathrm{CFT}^{7}$ can be expressed as

$$
Z_{c=47 / 2}=\left|f_{\mathrm{vac}}^{c=\frac{47}{2}}(\tau)\right|^{2}+\left|f_{\frac{3}{2}}^{c=\frac{47}{2}}(\tau)\right|^{2}+\left|f_{\frac{31}{16}}^{c=\frac{47}{2}}(\tau)\right|^{2} .
$$

Now, we add a discrete set of primaries having the conformal weights as below to the SDP problem:

$$
\begin{aligned}
&\{(\Delta, j) \mid \Delta=3+j, 5+j, 7+j, \cdots, 2 \delta+3+j\} \\
& \cup\{(\Delta, j) \mid \Delta=4+j, 6+j, 8+j, \cdots, 2 \delta+4+j\} \\
& \cup\left\{(\Delta, j) \mid \Delta=\frac{31}{8}+j, \frac{47}{8}+j, \frac{63}{8}+j, \cdots, 2 \delta+\frac{31}{8}+j\right\},
\end{aligned}
$$

for $0 \leq j \leq j_{\max }$ and an arbitrary positive integer $\delta$. For instance, the SDP problem with $\delta=10$ results in the maximal degeneracies of the extremal spectrum summarized in table 15. It is easy to show that the partition function (4.31) saturates these upper bounds. We thus expect that the putative CFT of our interest has the Baby Monster symmetry.

\footnotetext{
${ }^{7}$ The characters of the Baby Monster modules $\chi_{\mathrm{VB}_{(0,1,2)}^{\natural}}$ are given by

$$
j(\tau)-744=\chi_{\mathrm{VB}_{(0)}^{\natural}}(\tau) \chi_{\mathrm{vac}}^{\text {Ising }}(\tau)+\chi_{\mathrm{VB}_{(1)}^{\natural}}(\tau) \chi_{h=\frac{1}{2}}^{\text {Ising }}(\tau)+\chi_{\mathrm{VB}_{(3)}^{\natural}}(\tau) \chi_{h=\frac{1}{16}}^{\text {Ising }}(\tau),
$$

where $\chi_{h}^{\text {Ising }}(\tau)$ denote the Virasoro characters of the Ising model. From this, it is obvious that the ansatz (4.31) is invariant under the modular transformation.
} 


\begin{tabular}{|c|c|c|c|}
\hline$(h, \bar{h})$ & Max. Deg $(h, \bar{h})$ & $(h, \bar{h})$ & Max. Deg \\
\hline$\left(\frac{3}{2}, \frac{3}{2}\right)$ & 19105641.026984403127 & $\left(\frac{5}{2}, \frac{3}{2}\right)$ & 4980203754.2560961756 \\
\hline$\left(\frac{5}{2}, \frac{5}{2}\right)$ & 1298173112605.3499336 & $(2,2)$ & 9265025041.3227338031 \\
\hline$(3,2)$ & 919296372501.31519351 & $(3,3)$ & 91214629887092.699664 \\
\hline$\left(\frac{31}{16}, \frac{31}{16}\right)$ & 9265217540.6086142750 & $\left(\frac{47}{16}, \frac{31}{16}\right)$ & 1011288637613.8107313 \\
\hline
\end{tabular}

Table 15. This table summarizes the maximal degeneracies of the first few states in a gapped $c=47 / 2$ CFT. The maximum number of derivatives is set to $N_{\max }=65, \delta=10$, while the spin is truncated at $j_{\max }=40$.

\section{$5 \quad$ Bootstrapping with $\mathcal{W}$-algebra}

The two-dimensional CFTs with $\mathcal{W}\left(A_{2}\right)=\mathcal{W}(2,3)$ symmetry have been studied recently in $[23,24]$. To investigate the universal constraints on the spectrum of higher-spin "irrational" CFTs, i.e., $c>2$, the authors of [23, 24] apply the modular bootstrap method to the torus two-point function

$$
\operatorname{Tr}\left[W_{0}^{2} q^{L_{0}-\frac{c}{24}} \bar{q}^{\bar{L}_{0}-\frac{c}{24}}\right] .
$$

On the other hand, we will focus on the torus partition function

$$
Z(\tau, \bar{\tau})=\operatorname{Tr}\left[q^{L_{0}-\frac{c}{24}} \bar{q}^{\bar{L}_{0}-\frac{c}{24}}\right],
$$

under the assumption that there is a $\mathcal{W}\left(d_{1}, d_{2}, \ldots, d_{r}\right)$-algebra symmetry. This allows us to expand the partition function in the form

$$
\begin{aligned}
Z(\tau, \bar{\tau})= & \chi_{0}(\tau) \bar{\chi}_{0}(\bar{\tau})+\sum_{h, \bar{h}} d_{h, \bar{h}}\left[\chi_{h}(\tau) \bar{\chi}_{\bar{h}}(\bar{\tau})+\chi_{\bar{h}}(\tau) \bar{\chi}_{h}(\bar{\tau})\right] \\
& +\sum_{j=1} d_{j}\left[\chi_{j}(\tau) \bar{\chi}_{0}(\bar{\tau})+\chi_{0}(\tau) \bar{\chi}_{j}(\bar{\tau})\right]
\end{aligned}
$$

where the characters are given by (assuming the non-vacuum module is non-degenerate)

$$
\chi_{0}(\tau)=\frac{q^{-\frac{c-r}{24}}}{\eta(\tau)^{r}} \prod_{i=1}^{r} \prod_{j=1}^{d_{i}-1}\left(1-q^{j}\right), \quad \chi_{h}(\tau)=\frac{q^{h-\frac{c-r}{24}}}{\eta(\tau)^{r}} .
$$

In this section, we examine how the modular invariance for the theories with various $\mathcal{W}$-symmetries constrains the spectrum.

\section{$5.1 \quad$ Numerical bounds}

In this subsection, we examine modular constraints on the scalar gap $\Delta_{s}$, overall gap $\Delta_{o}$ and twist gap $\Delta_{t}$ for CFTs with $\mathcal{W}$-symmetry. To this end, we use the $\mathcal{W}$-algebra characters given in (2.35) and (2.36) to decompose the modular invariant partition function (3.2).

It turns out that the numerical upper bounds with $\mathcal{W}\left(d_{1}, d_{2}, \cdots, d_{r}\right)$-algebra exhibit the same bounds as in the case for the Virasoro algebra except for the region $c \lesssim r$. For 


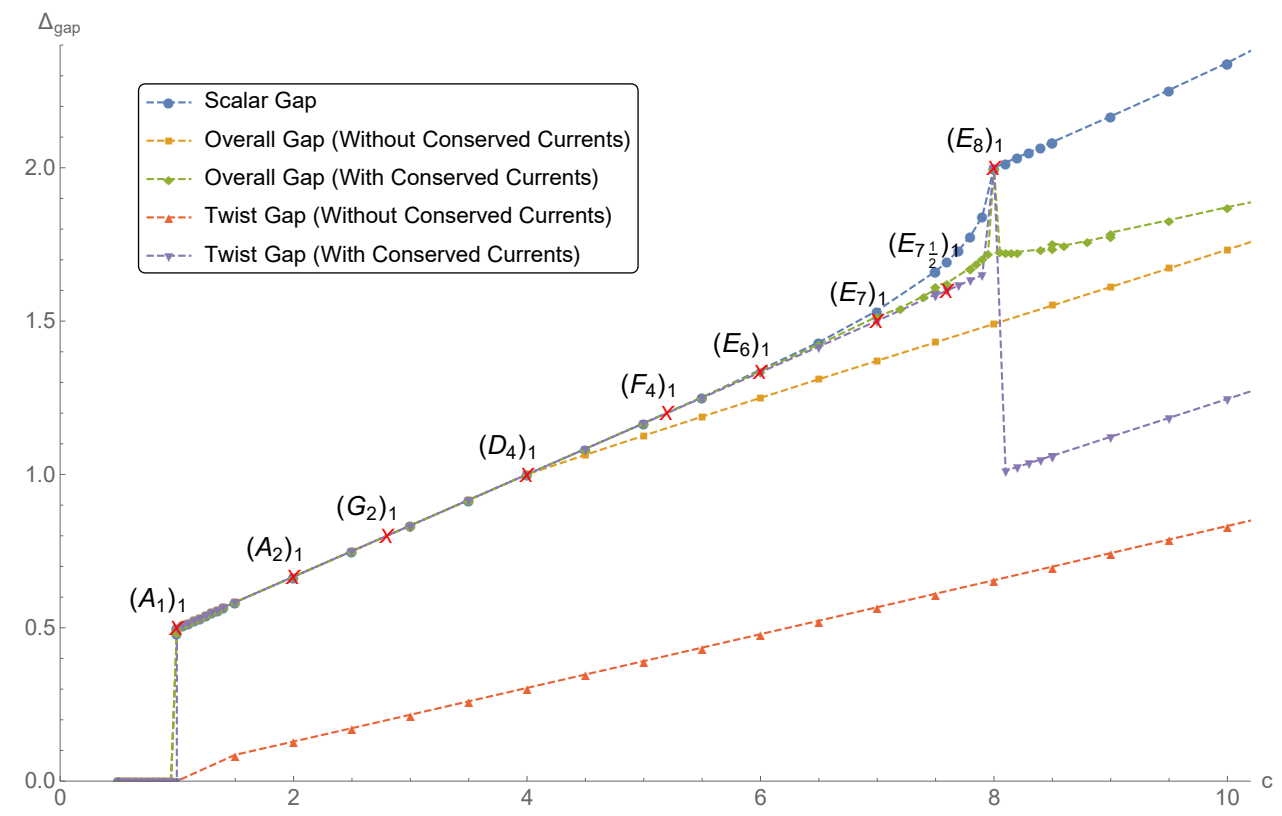

Figure 16. Numerical upper bounds on the scalar gap, overall gap and twist gap with $\mathcal{W}\left(F_{4}\right)=\mathcal{W}(2,6,8,12)$ algebra in the range of $1 \leq c \leq 10$.

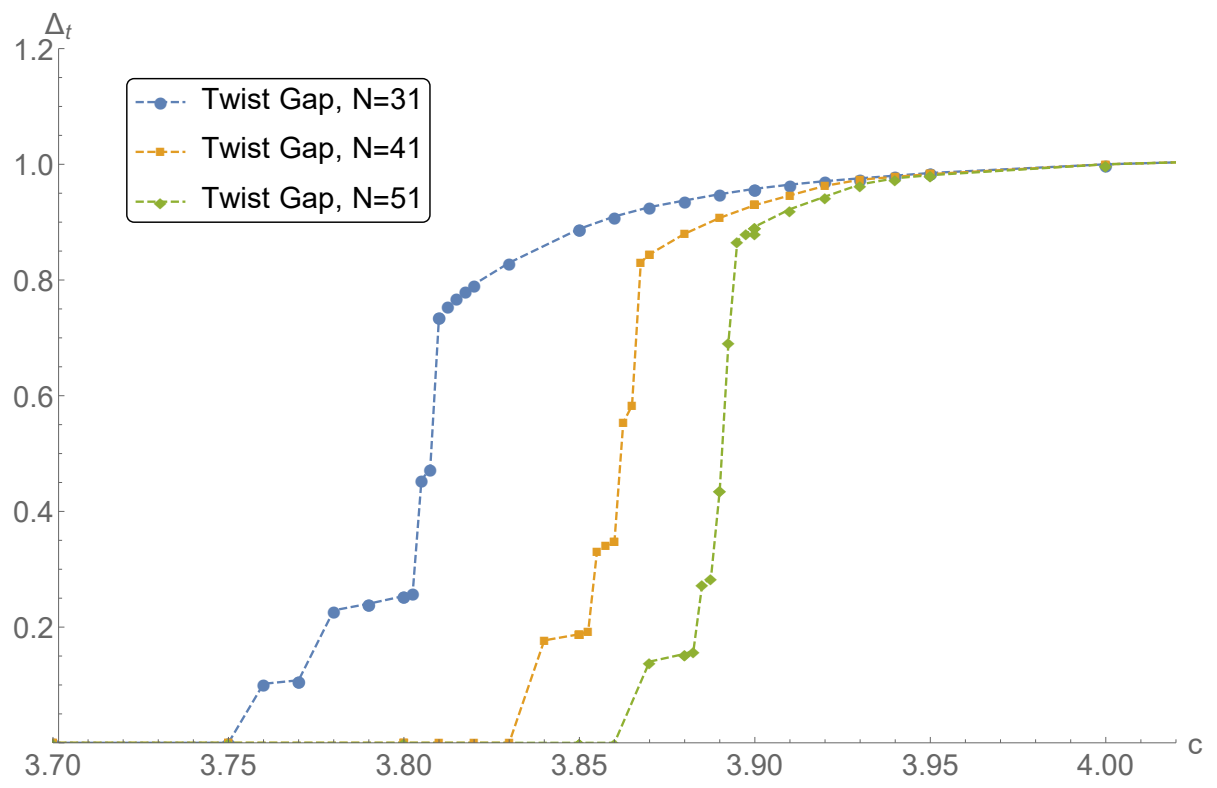

Figure 17. Numerical upper bounds on twist gap with $\mathcal{W}\left(F_{4}\right)=\mathcal{W}(2,6,8,12)$ algebra in the range of $3.7 \leq c \leq 4.0$. The sharp cliff is pushed towards $c=4$ as the number of derivatives gets increased.

instance, we summarize the numerical results with the $\mathcal{W}\left(F_{4}\right)=\mathcal{W}(2,6,8,12)$-algebra in figure 16. Unlike the case of the Virasoro symmetry, the numerical bounds sharply rise near $c \sim 4$. As is depicted in figure 17, the sharp cliff is pushed towards $c=4$ as we increase the total number of derivatives $N_{\max }$. We further investigate how the position of 


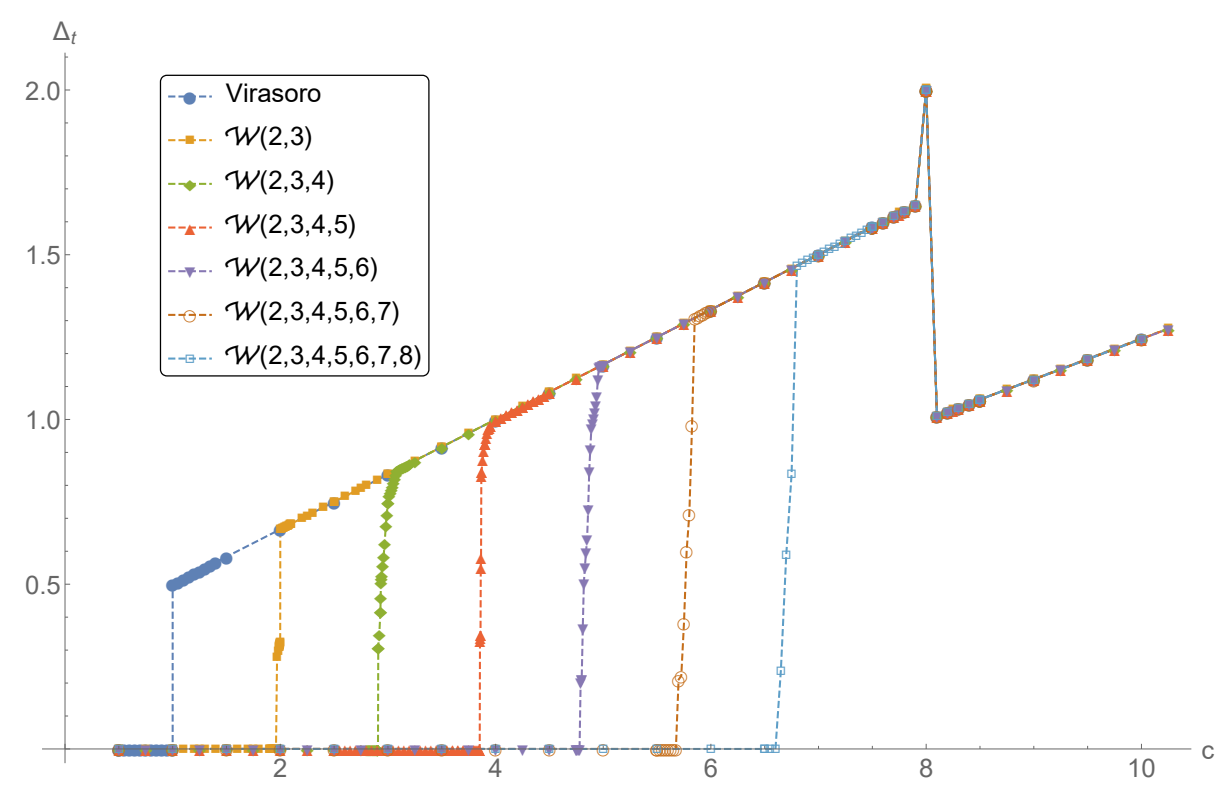

Figure 18. Numerical bounds on twist gap with various $\mathcal{W}$-algebra. We assume the presence of the conserved currents of $j \geq 1$ in the spectrum.

the sharp cliff depends on the rank of the $\mathcal{W}$-algebra. As shown in figure 18, these values are placed near the rank of the corresponding $\mathcal{W}$-algebras. Based on these observations, we can legitimize the assumption in (2.35) and (2.36) that the unitary irreducible representations of $\mathcal{W}\left(d_{1}, d_{2}, \cdots, d_{r}\right)$-algebra do not contain any nontrivial null states when $c \geq r$.

We also find that the $\widehat{\mathfrak{g}}_{k=1}$ WZW model can be placed at the numerical upper bound on $\Delta_{t}$ for the $\mathcal{W}(\mathfrak{g})$-algebra. To illustrate this, let us consider the numerical bounds for the $\mathcal{W}\left(A_{2}\right)=\mathcal{W}(2,3)$ and $\mathcal{W}\left(G_{2}\right)=\mathcal{W}(2,6)$. Figure 19 shows that the $\left(\widehat{A}_{2}\right)_{1}$ WZW model indeed sits at the numerical bound for the $\mathcal{W}(2,3)$-algebra as expected. However, we notice that the model violates the modular constraint for the $\mathcal{W}(2,6)$-algebra. This is because when we decompose the partition function of the $\left(\widehat{A}_{2}\right)_{1}$ WZW model $(2.17)$ (with $h=\frac{1}{3}$ and $\left.N\left(\widehat{\mathfrak{g}}_{1}\right)=1\right)$ using the $\mathcal{W}(2,6)$ characters, some of the multiplicities $d_{h, \bar{h}}$ become negative.

Figure 20 shows the numerical bound on $\Delta_{t}$ for the $\mathcal{W}\left(A_{3}\right)=\mathcal{W}(2,3,4)$-algebra. Again, $\left(\widehat{A}_{3}\right)_{1}$ WZW model is located at $\left(c=3, \Delta_{t}=\frac{3}{4}\right)$ on the numerical upper bound. Later we verify that the spectrum of $\left(\widehat{A}_{3}\right)_{1}$ WZW model maximizes the degeneracies of the extremal spectrum for $c=3$ and $\Delta_{t}=\frac{3}{4}$. It is interesting to see that the $\left(\widehat{A}_{3}\right)_{1} \mathrm{WZW}$ model is realized at the numerical boundary only using the $\mathcal{W}(2,3,4)$-algebra, but not using the other $\mathcal{W}$-algebras (including Virasoro). Note that $A_{3}$ does not belong to the Deligne's exceptional series.

\subsection{Spectral analysis}

\section{- Spectrum analysis for the $\left(\widehat{F}_{4}\right)_{1}$ WZW}

Let us show that the $\widehat{\mathfrak{g}}_{k=1}$ WZW model saturates the upper bound not only on $\Delta_{t}$ but also on the degeneracies of the extremal spectrum for the $\mathcal{W}(\mathfrak{g})$-algebra. As an example, let us consider a hypothetical CFT with $c=\frac{26}{5}$ and $\Delta_{t}=\frac{6}{5}$ which lies at the numerical boundary on $\Delta_{t}$ for the $\mathcal{W}\left(F_{4}\right)=\mathcal{W}(2,6,8,12)$-algebra. 


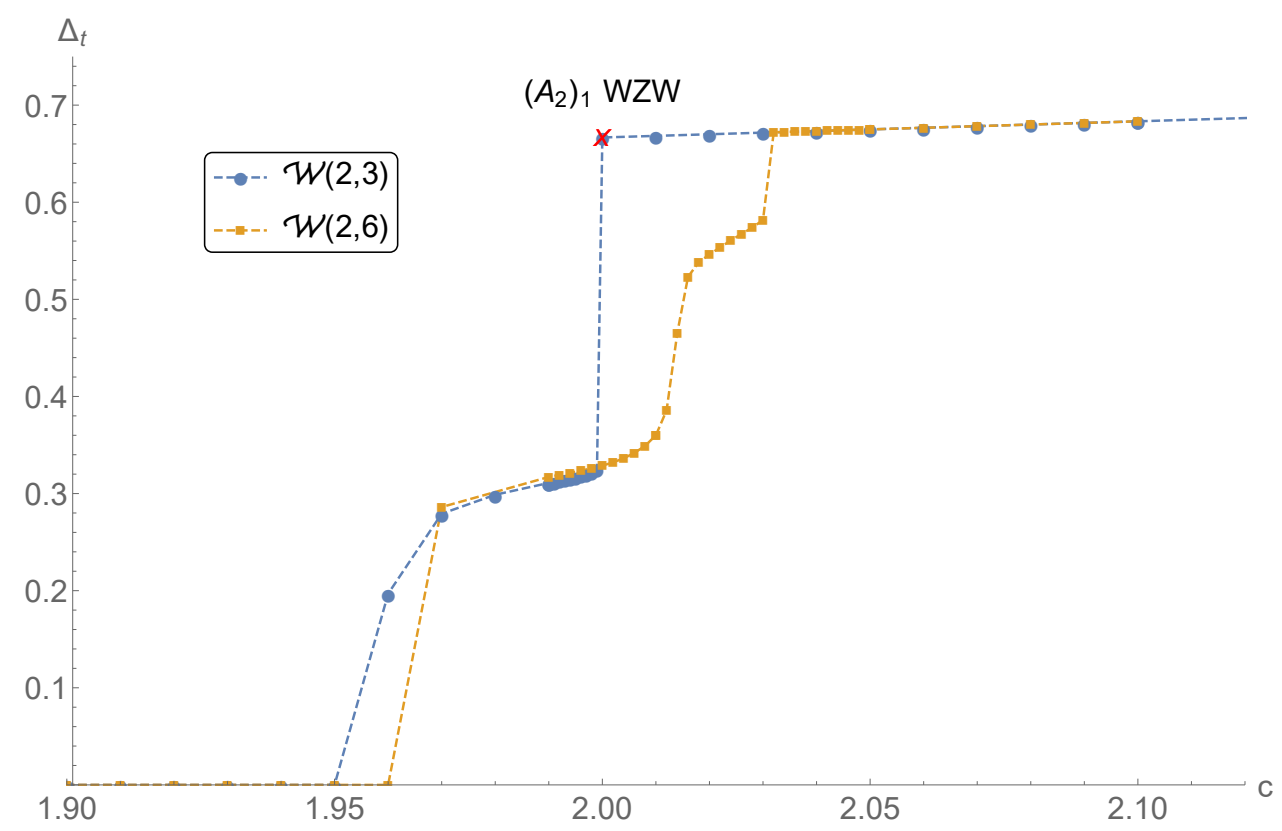

Figure 19. Numerical bounds on the twist gap for $\mathcal{W}(2,3)$ and $\mathcal{W}(2,6)$-algebra. The upper bound obtained from the $\mathcal{W}(2,3)$-algebra realizes the $\left(A_{2}\right)_{1}$ WZW model at the numerical boundary, while $\mathcal{W}(2,6)$ does not.
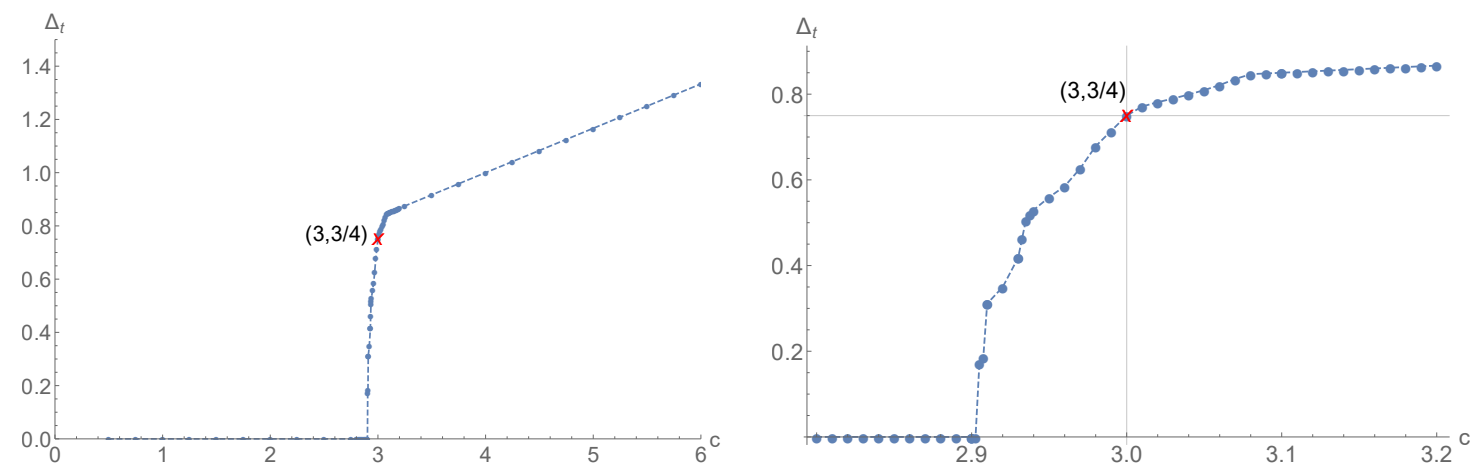

Figure 20. Numerical bound on twist gap with $\mathcal{W}(2,3,4)$ algebra. It turns out that the $\left(\widehat{A}_{3}\right)_{1}$ WZW model is realized on the numerical boundary.

To study the extremal spectrum of a hypothetical CFT, we apply the EFM analysis. The conformal dimensions of spin- 0 and spin-1 extremal spectrum can be read off from figure 21 , given by $\Delta_{j}=\left\{\frac{6}{5}+j+2 n, 2+j+2 n\right\}$ for $j=0,1$ and $n \in \mathbb{Z}_{n \geq 0}$. The maximal degeneracies of various primaries in the extremal spectrum are summarized in table 16. It implies that the partition function of the putative CFT of our interest can be expressed as follow,

$$
\begin{aligned}
Z_{c=\frac{26}{5}}^{\mathcal{W}(2,6,8,12)}(q, \bar{q})= & \chi_{0}(\tau) \bar{\chi}_{0}(\bar{\tau})+676 \chi_{\frac{3}{5}}(\tau) \bar{\chi}_{\frac{3}{5}}(\bar{\tau})+5070\left(\chi_{\frac{3}{5}}(\tau) \bar{\chi}_{\frac{8}{5}}(\bar{\tau})+\text { c.c. }\right) \\
& +2704 \chi_{1}(\tau) \bar{\chi}_{1}(\bar{\tau})+8736\left(\chi_{2}(\tau) \bar{\chi}_{1}(\bar{\tau})+\text { c.c. }\right) \\
& \left.+52\left(\chi_{1}(\tau) \bar{\chi}_{0}(\bar{\tau})+\text { c.c. }\right)\right)+168\left(\chi_{2}(\tau) \bar{\chi}_{0}(\bar{\tau})+\text { c.c. }\right)+\cdots,
\end{aligned}
$$



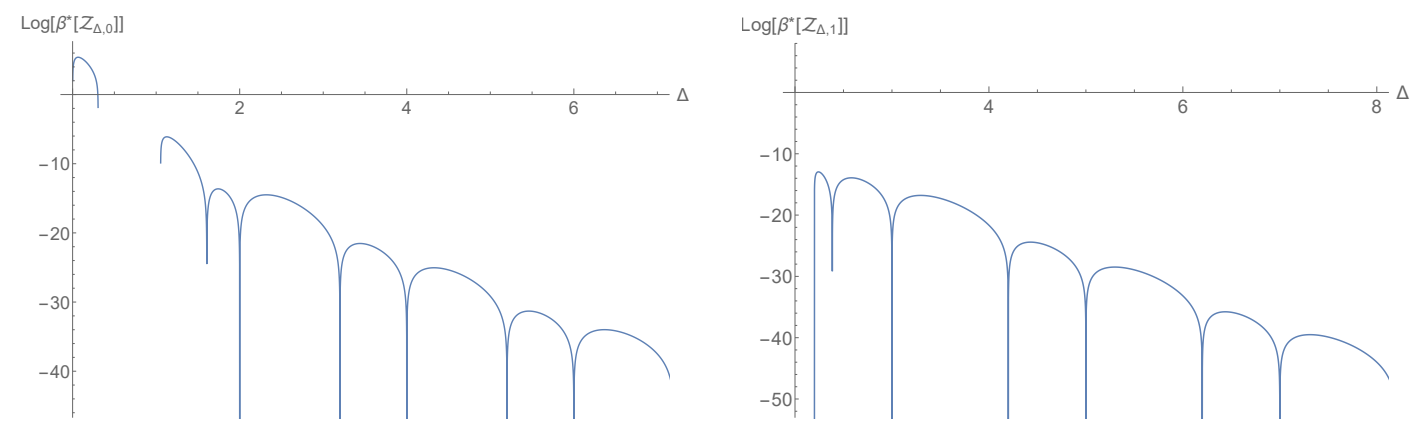

Figure 21. The Extremal Functional Method applied for $\mathcal{W}(2,6,8,12), c=\frac{26}{5}$. In this plot, we set $N_{\max }=51$ and $j_{\max }=30$. The left figure is the spin-0 sector, while the right one is the spin- 1 sector.

\begin{tabular}{|c|c|c|c|c|c|}
\hline$(h, \bar{h})$ & Max. Deg & $(h, \bar{h})$ & Max. Deg & $(h, \bar{h})$ & Max. Deg \\
\hline$\left(\frac{3}{5}, \frac{3}{5}\right)$ & 676.00000 & $(1,1)$ & 2704.0000 & $(1,0)$ & 52.00000 \\
\hline$\left(\frac{3}{5}, \frac{8}{5}\right)$ & 5070.0000 & $(2,1)$ & 8736.000 & $(2,0)$ & 168.0000 \\
\hline$\left(\frac{3}{5}, \frac{13}{5}\right)$ & 14508.000 & $(3,1)$ & 29900.000 & $(3,0)$ & 575.0000 \\
\hline$\left(\frac{8}{5}, \frac{8}{5}\right)$ & 38025.000 & $(2,2)$ & 28224.000 & $(4,0)$ & 1118.0000 \\
\hline$\left(\frac{8}{5}, \frac{13}{5}\right)$ & 108810.00 & $(2,3)$ & 96600.008 & $(5,0)$ & 2700.0000 \\
\hline$\left(\frac{13}{5}, \frac{13}{5}\right)$ & 311364.001 & $(3,3)$ & 330625.00 & $(6,0)$ & 4780.0000 \\
\hline
\end{tabular}

Table 16. Degeneracies of the first few states in $c=\frac{26}{5}$ CFT. We set $N_{\max }=55$ and $j_{\max }=40$. We used the $\mathcal{W}(2,6,8,12)$ character with $c=\frac{26}{5}$.
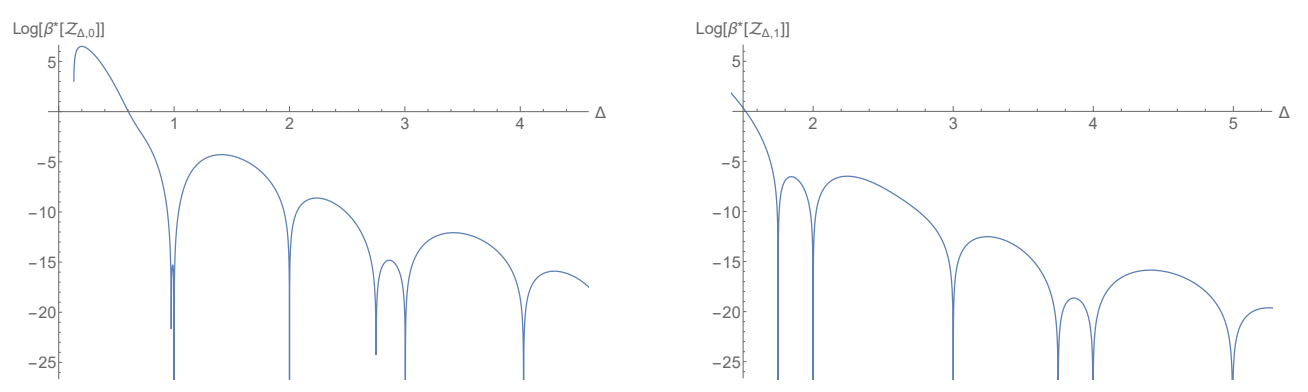

Figure 22. Extremal Functional Method applied to $c=3$ CFT with $\mathcal{W}(2,3,4)$-algebra. In this plot, we set $N_{\max }=51$ and $j_{\max }=30$.

where $\chi_{h}$ denotes a $\mathcal{W}(2,6,8,12)$ character. One can easily show that (5.5) agrees with the partition function of the $\left(\widehat{F}_{4}\right)_{1}$ WZW model (4.13).

- Spectrum analysis for the $\left(\widehat{A}_{3}\right)_{1} W Z W$

Let us discuss the point $\left(c=3, \Delta_{t}=\frac{3}{4}\right.$ ) placed at the numerical boundary on $\Delta_{t}$ for the $\mathcal{W}\left(A_{3}\right)=\mathcal{W}(2,3,4)$-algebra. The extremal spectrum at this point and their maximal degeneracies are illustrated in figure 22 and table 17. From these results, the conformal dimensions of spin- 0 and spin- 1 primaries can be obtained as $\Delta_{j=0}=\left\{\frac{3}{4}+2 n, 1+n\right\}$ and $\Delta_{j=1}=\left\{\frac{7}{4}+2 n, 2+n\right\}$, for $n \in \mathbb{Z}_{n \geq 0}$. 


\begin{tabular}{|c|c|c|c|c|c|}
\hline$(h, \bar{h})$ & Max. Deg & $(h, \bar{h})$ & Max. Deg & $(h, \bar{h})$ & Max. Deg \\
\hline$\left(\frac{3}{8}, \frac{3}{8}\right)$ & 32.00000 & $\left(\frac{1}{2}, \frac{1}{2}\right)$ & 36.000000 & $(1,1)$ & 225.25714 \\
\hline$\left(\frac{3}{8}, \frac{11}{8}\right)$ & 96.00000 & $\left(\frac{1}{2}, \frac{3}{2}\right)$ & 48.00000 & $(1,2)$ & 75.00020 \\
\hline$\left(\frac{11}{8}, \frac{11}{8}\right)$ & 288.01585 & $\left(\frac{3}{2}, \frac{3}{2}\right)$ & 64.11818 & $(2,2)$ & 25.00500 \\
\hline
\end{tabular}

Table 17. Here $N_{\max }=51, j_{\max }=30$. We used the $\mathcal{W}(2,3,4)$ character with $c=3$.

We can recast the partition function of a CFT containing the primaries in table 17 as the following form

$$
Z_{A_{3}}^{\mathcal{W}(2,3,4)}(q, \bar{q})=\left|f_{\text {vac }}^{c=3}(q)\right|^{2}+\left|f_{\frac{1}{2}}^{c=3}(q)\right|^{2}+2\left|f_{\frac{3}{8}}^{c=3}(q)\right|^{2},
$$

where $f_{\text {vac }}^{c=3}(q), f_{\frac{1}{2}}^{c=3}(q)$ and $f_{\frac{3}{8}}^{c=3}(q)$ are $\left(\widehat{A}_{3}\right)_{1}$ characters given by,

$$
\begin{aligned}
& f_{\text {vac }}^{c=3}(q)=\chi_{[1 ; 0,0,0]}(q)=q^{-\frac{1}{8}}\left(1+15 q+51 q^{2}+172 q^{3}+453 q^{4}+\mathcal{O}\left(q^{5}\right)\right), \\
& f_{\frac{1}{2}}^{c=3}(q)=\chi_{[0 ; 0,1,0]}(q)=q^{\frac{1}{2}-\frac{1}{8}}\left(6+26 q+102 q^{2}+276 q^{3}+728 q^{4}+\mathcal{O}\left(q^{5}\right)\right), \\
& f_{\frac{3}{8}}^{c=3}(q)=\chi_{[0 ; 1,0,0]}(q)=\chi_{[0 ; 0,0,1]}(q)=q^{\frac{3}{8}-\frac{1}{8}}\left(4+24 q+84 q^{2}+248 q^{3}+648 q^{4}+\mathcal{O}\left(q^{5}\right)\right) .
\end{aligned}
$$

(5.6) is nothing but the partition function of the $\left(\widehat{A}_{3}\right)_{1}$ WZW model. We thus identify a putative CFT of our interest with $c=3$ as the $\left(\widehat{A}_{3}\right)_{1}$ WZW model.

\subsection{Accumulation of the spectrum}

Figure 23 shows the plots of the maximal degeneracy versus the conformal dimension of the lowest primary in a theory with $\mathcal{W}\left(d_{1}, d_{2}, \cdots, d_{r}\right)$ symmetry at various central charges. We notice that there is no upper bound on the degeneracy as the conformal dimension $\Delta_{t}$ approaches to $\frac{c-r}{12}$. We also observe that the location at which the degeneracy diverge is independent of the presence of holomorphic/anti-holomorphic currents other than $\mathcal{W}$ symmetry. The divergence can be explained from the fact that infinitely many primaries get accumulated at $h=\frac{c-r}{24}[14,23]$. For the self-containment, we briefly present the derivation of $[14,23]$ below.

In order to understand the origin of the divergence, it is sufficient to consider the theories without conserved currents. In the limit $\bar{\tau} \rightarrow i \infty$, the vacuum character of $\mathcal{W}$ algebra dominates the partition function:

$$
\lim _{\bar{\tau} \rightarrow-i \infty}\left[\frac{Z(\tau, \bar{\tau})}{\bar{\chi}_{0}(\bar{\tau})}\right]=\chi_{0}(\tau)
$$

We further consider the limit $\tau \rightarrow i 0^{+}$where the vacuum character behaves as

$$
\lim _{\tau \rightarrow i 0^{+}} \chi_{0}(\tau)=\lim _{\tau \rightarrow i 0^{+}}\left(q^{\prime}\right)^{-\frac{r}{24}}
$$




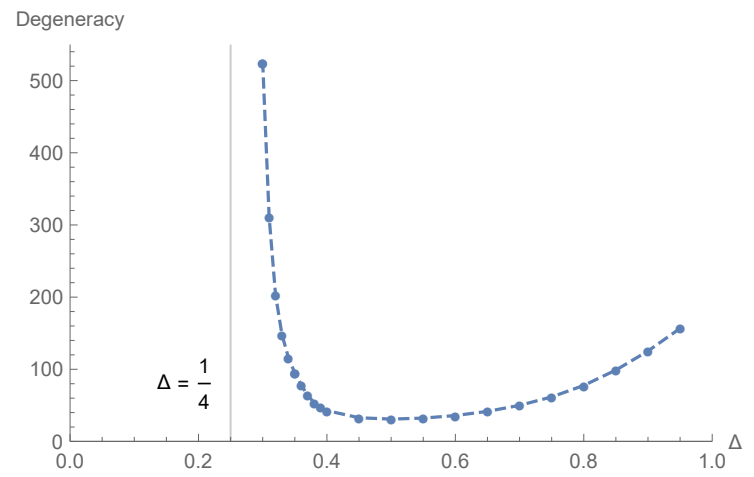

(a) $c=4$, Virasoro algebra

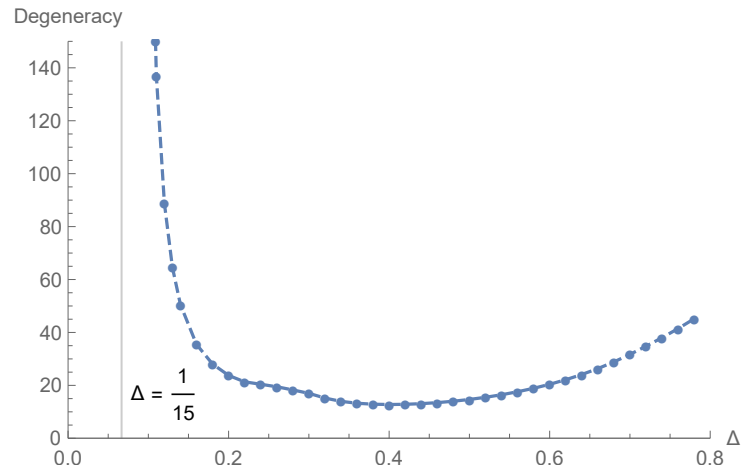

(c) $c=\frac{14}{5}, \mathcal{W}(2,3)$-algebra

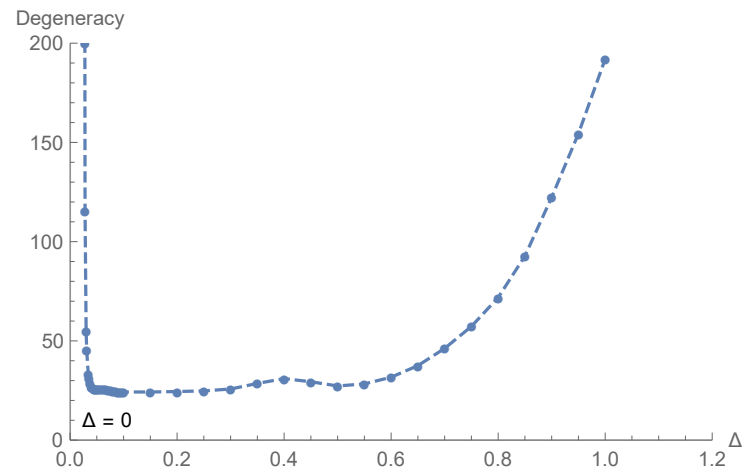

(e) $c=4, \mathcal{W}(2,6,8,12)$-algebra

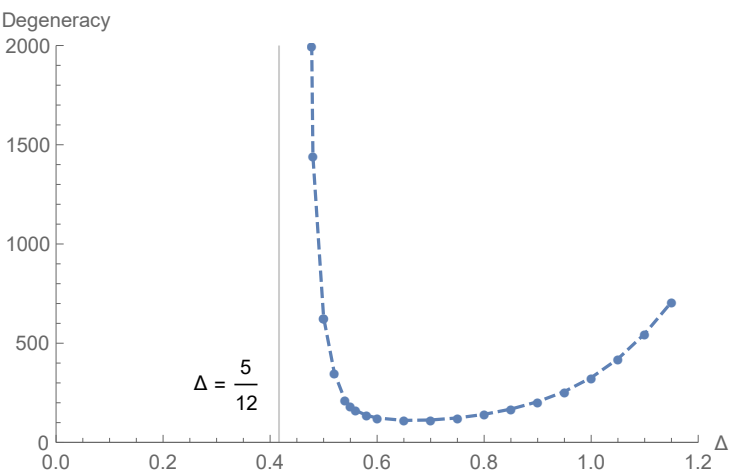

(b) $c=6$, Virasoro algebra

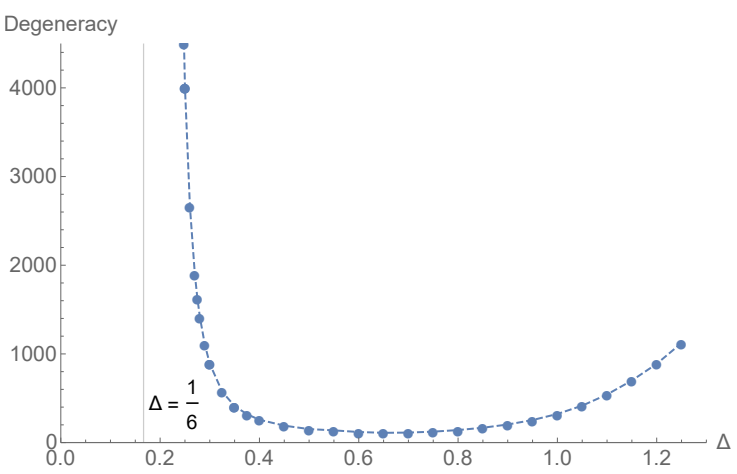

(d) $c=6, \mathcal{W}(2,6,8,12)$-algebra

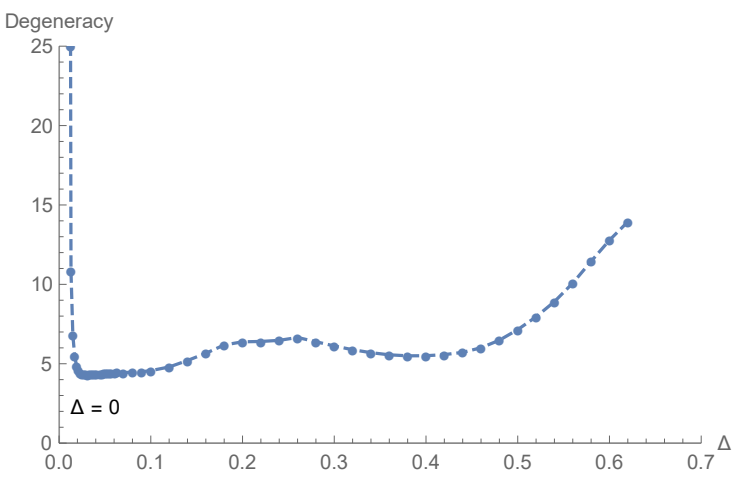

(f) $c=2, \mathcal{W}(2,3)$-algebra

Figure 23. The maximal degeneracies of the lowest primary with various $\mathcal{W}$-algebras and central charges.

where $q^{\prime} \equiv e^{-\frac{2 \pi i}{\tau}}$. As a consequence, the modular invariance of the partition function $Z(\tau, \bar{\tau})=Z(-1 / \tau,-1 / \bar{\tau})$ in the above special limits requires

$$
\lim _{\tau \rightarrow i 0^{+}} e^{\frac{2 \pi i}{24 \tau} r}=\lim _{\tau \rightarrow i 0^{+}} \lim _{\bar{\tau} \rightarrow-i \infty}\left[\chi_{0}\left(-\frac{1}{\tau}\right) \cdot \frac{\bar{\chi}_{0}\left(-\frac{1}{\bar{\tau}}\right)}{\bar{\chi}_{0}(\bar{\tau})}+\sum_{h, \bar{h}} d_{h, \bar{h}} \chi_{h}\left(-\frac{1}{\tau}\right) \cdot \frac{\bar{\chi}_{\bar{h}}\left(-\frac{1}{\bar{\tau}}\right)}{\bar{\chi}_{0}(\bar{\tau})}\right] .
$$

Note that $\lim _{\bar{\tau} \rightarrow-i \infty} \frac{\bar{\chi}_{\bar{h}}\left(-\frac{1}{\bar{\tau}}\right)}{\overline{\bar{\chi}} 0(\bar{\tau})}=\lim _{\bar{\tau} \rightarrow-i \infty}(\bar{q})^{(c-r) / 24} \rightarrow 0$ for $c>r$. If the limit and the 
summation in the r.h.s. of (5.10) were to commute, we would therefore say that the r.h.s. of (5.10) vanish, which is inconsistent with the l.h.s. of (5.10). One can resolve the above contradiction only when there exist infinitely many primaries of weight $h$ accumulating to $\frac{c-r}{24}$. The infinite degeneracy at $h=\frac{c-r}{24}$ and $\bar{h}=\frac{c-r}{24}$ then explains the divergence behavior in figure 23 .

\section{Acknowledgments}

We would like to thank Jeffrey Harvey, Sunil Mukhi, Kimyeong Lee and Soo-Jong Rey for useful discussions. We thank KIAS Center for Advanced Computation for providing computing resources. The research of S.L. is supported in part by the National Research Foundation of Korea (NRF) Grant NRF-2017R1C1B1011440. S.L. and J.S. thank the organizers of the Pollica Summer Workshop 2017 for the hospitality, and was partly supported by the ERC STG grant 306260 during the Pollica Summer Workshop. We also thank the organizers of the workshop "Geometry of String and Gauge Theories" at CERN, and also CERN-Korea Theory Collaboration funded by National Research Foundation (Korea) for the hospitality and support. J.S. also thanks the organizers of the Summer Simons Workshops in Mathematics and Physics 2017 for the hospitality.

Open Access. This article is distributed under the terms of the Creative Commons Attribution License (CC-BY 4.0), which permits any use, distribution and reproduction in any medium, provided the original author(s) and source are credited.

\section{References}

[1] A.A. Belavin, A.M. Polyakov and A.B. Zamolodchikov, Infinite Conformal Symmetry in Two-Dimensional Quantum Field Theory, Nucl. Phys. B 241 (1984) 333 [InSPIRE].

[2] G.W. Moore and N. Seiberg, Classical and Quantum Conformal Field Theory, Commun. Math. Phys. 123 (1989) 177 [INSPIRE].

[3] R. Rattazzi, V.S. Rychkov, E. Tonni and A. Vichi, Bounding scalar operator dimensions in 4D CFT, JHEP 12 (2008) 031 [arXiv:0807.0004] [INSPIRE].

[4] D. Poland, D. Simmons-Duffin and A. Vichi, Carving Out the Space of $4 D$ CFTs, JHEP 05 (2012) 110 [arXiv:1109.5176] [inSPIRE].

[5] C.-M. Chang and Y.-H. Lin, Bootstrapping 2D CFTs in the Semiclassical Limit, JHEP 08 (2016) 056 [arXiv : 1510.02464] [INSPIRE].

[6] H. Kim, P. Kravchuk and H. Ooguri, Reflections on Conformal Spectra, JHEP 04 (2016) 184 [arXiv: 1510.08772] [INSPIRE].

[7] C.-M. Chang and Y.-H. Lin, Bootstrap, universality and horizons, JHEP 10 (2016) 068 [arXiv: 1604.01774] [INSPIRE].

[8] Y.-H. Lin, S.-H. Shao, D. Simmons-Duffin, Y. Wang and X. Yin, $\mathcal{N}=4$ superconformal bootstrap of the K3 CFT, JHEP 05 (2017) 126 [arXiv: 1511.04065] [INSPIRE].

[9] Y.-H. Lin, S.-H. Shao, Y. Wang and X. Yin, $(2,2)$ superconformal bootstrap in two dimensions, JHEP 05 (2017) 112 [arXiv:1610.05371] [INSPIRE]. 
[10] J.-B. Bae, K. Lee and S. Lee, Bootstrapping Pure Quantum Gravity in AdS3, arXiv: 1610.05814 [INSPIRE].

[11] S. Hellerman, A Universal Inequality for CFT and Quantum Gravity, JHEP 08 (2011) 130 [arXiv: 0902.2790] [INSPIRE].

[12] D. Friedan and C.A. Keller, Constraints on 2d CFT partition functions, JHEP 10 (2013) 180 [arXiv:1307.6562] [INSPIRE].

[13] T. Hartman, C.A. Keller and B. Stoica, Universal Spectrum of 2d Conformal Field Theory in the Large c Limit, JHEP 09 (2014) 118 [arXiv:1405.5137] [INSPIRE].

[14] S. Collier, Y.-H. Lin and X. Yin, Modular Bootstrap Revisited, arXiv:1608.06241 [INSPIRE].

[15] S. Hellerman and C. Schmidt-Colinet, Bounds for State Degeneracies in 2D Conformal Field Theory, JHEP 08 (2011) 127 [arXiv:1007.0756] [INSPIRE].

[16] C.A. Keller and H. Ooguri, Modular Constraints on Calabi-Yau Compactifications, Commun. Math. Phys. 324 (2013) 107 [arXiv: 1209.4649] [InSPIRE].

[17] J.D. Qualls and A.D. Shapere, Bounds on Operator Dimensions in 2D Conformal Field Theories, JHEP 05 (2014) 091 [arXiv: 1312.0038] [INSPIRE].

[18] J.D. Qualls, Universal Bounds in Even-Spin CFTs, JHEP 12 (2015) 001 [arXiv:1412.0383] [INSPIRE].

[19] J.D. Qualls, Universal Bounds on Operator Dimensions in General 2D Conformal Field Theories, arXiv: 1508.00548 [INSPIRE].

[20] J. Cardy, A. Maloney and H. Maxfield, A new handle on three-point coefficients: OPE asymptotics from genus two modular invariance, JHEP 10 (2017) 136 [arXiv:1705.05855] [INSPIRE].

[21] C.A. Keller, G. Mathys and I.G. Zadeh, Bootstrapping Chiral CFTs at Genus Two, arXiv: 1705.05862 [INSPIRE].

[22] M. Cho, S. Collier and X. Yin, Genus Two Modular Bootstrap, arXiv:1705.05865 [inSPIRE].

[23] N. Afkhami-Jeddi, K. Colville, T. Hartman, A. Maloney and E. Perlmutter, Constraints on Higher Spin $\mathrm{CFT}_{2}$, arXiv: 1707.07717 [INSPIRE].

[24] L. Apolo, Bounds on CFTs with $\mathcal{W}_{3}$ algebras and AdS $S_{3}$ higher spin theories, Phys. Rev. D 96 (2017) 086003 [arXiv: 1705.10402] [INSPIRE].

[25] J.D. Brown and M. Henneaux, Central Charges in the Canonical Realization of Asymptotic Symmetries: An Example from Three-Dimensional Gravity, Commun. Math. Phys. 104 (1986) 207 [INSPIRE].

[26] D. Simmons-Duffin, A Semidefinite Program Solver for the Conformal Bootstrap, JHEP 06 (2015) 174 [arXiv: 1502. 02033] [INSPIRE].

[27] N. Benjamin, E. Dyer, A.L. Fitzpatrick and S. Kachru, Universal Bounds on Charged States in $2 d$ CFT and 3d Gravity, JHEP 08 (2016) 041 [arXiv: 1603.09745] [INSPIRE].

[28] S.D. Mathur, S. Mukhi and A. Sen, On the Classification of Rational Conformal Field Theories, Phys. Lett. B 213 (1988) 303 [INSPIRE].

[29] H.R. Hampapura and S. Mukhi, On 2d Conformal Field Theories with Two Characters, JHEP 01 (2016) 005 [arXiv: 1510.04478] [INSPIRE]. 
[30] I.B. Frenkel, J. Lepowsky and A. Meurman, A natural representation of the fischer-griess monster with the modular function j as character, Proc. Nat. Acad. Sci. 81 (1984) 3256.

[31] G. Hoehn, Selbstduale vertexoperatorsuperalgebren und das babymonster (self-dual vertex operator super algebras and the baby monster), arXiv:0706.0236.

[32] M. Henneaux and S.-J. Rey, Nonlinear $W_{\infty}$ as Asymptotic Symmetry of Three-Dimensional Higher Spin Anti-de Sitter Gravity, JHEP 12 (2010) 007 [arXiv: 1008.4579] [INSPIRE].

[33] S.D. Mathur, S. Mukhi and A. Sen, Reconstruction of Conformal Field Theories From Modular Geometry on the Torus, Nucl. Phys. B 318 (1989) 483 [InSPIRE].

[34] T. Gannon, WZW commutants, lattices and level 1 partition functions, Nucl. Phys. B 396 (1993) 708 [hep-th/9209043] [INSPIRE].

[35] M.P. Tuite, Exceptional Vertex Operator Algebras and the Virasoro Algebra, Contemp. Math. 497 (2009) 213 [arXiv:0811.4523] [INSPIRE].

[36] H.R. Hampapura and S. Mukhi, Two-dimensional RCFT's without Kac-Moody symmetry, JHEP 07 (2016) 138 [arXiv: 1605.03314] [INSPIRE].

[37] I. Frenkel, J. Lepowsky and A. Meurman, Vertex operator algebras and the monster, Academic Press (1989).

[38] P. Bouwknegt and K. Schoutens, W symmetry in conformal field theory, Phys. Rept. 223 (1993) 183 [hep-th/9210010] [INSPIRE].

[39] A.B. Zamolodchikov, Infinite Additional Symmetries in Two-Dimensional Conformal Quantum Field Theory, Theor. Math. Phys. 65 (1985) 1205 [INSPIRE].

[40] N.J. Iles and G.M.T. Watts, Characters of the $W_{3}$ algebra, JHEP 02 (2014) 009 [arXiv: 1307.3771] [INSPIRE].

[41] N.J. Iles and G.M.T. Watts, Modular properties of characters of the $W_{3}$ algebra, JHEP 01 (2016) 089 [arXiv:1411.4039] [INSPIRE].

[42] R. Blumenhagen, M. Flohr, A. Kliem, W. Nahm, A. Recknagel and R. Varnhagen, $W$ algebras with two and three generators, Nucl. Phys. B 361 (1991) 255 [INSPIRE].

[43] V.G. Drinfeld and V.V. Sokolov, Lie algebras and equations of Korteweg-de Vries type, J. Sov. Math. 30 (1984) 1975 [inSPIRE].

[44] M. Bershadsky and H. Ooguri, Hidden SL(n) Symmetry in Conformal Field Theories, Commun. Math. Phys. 126 (1989) 49 [INSPIRE].

[45] B. Feigin and E. Frenkel, Quantization of the Drinfeld-Sokolov reduction, Phys. Lett. B 246 (1990) 75 [INSPIRE].

[46] S. El-Showk and M.F. Paulos, Bootstrapping Conformal Field Theories with the Extremal Functional Method, Phys. Rev. Lett. 111 (2013) 241601 [arXiv:1211.2810] [INSPIRE].

[47] J.M. Landsberg and L. Manivel, The sextonions and $E_{7 \frac{1}{2}}$, Adv. Math. 201 (2006) 143 [math/0402157].

[48] GAP Group, GAP - Groups, Algorithms and Programming, Version 4.8.7 (2017), https://www.gap-system.org/. 